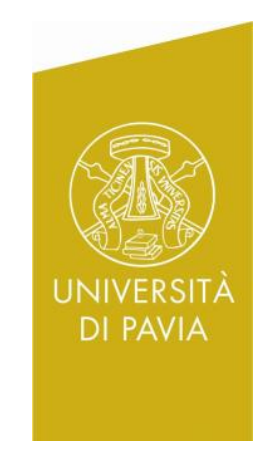

Department of Economics and Management DEM Working Paper Series

\title{
Forecasting dynamically asymmetric fluctuations of the U.S. business cycle.
}

Emilio Zanetti Chini

(Università di Pavia)

\# $156(03-18)$

Via San Felice, 5

I-27100 Pavia

economiaweb.unipv.it

March 2018 


\title{
Forecasting dynamically asymmetric fluctuations of the U.S. business cycle.
}

\author{
EMilio ZanetTi Chini \\ University of Pavia \\ Department of Economics and Management \\ Via San Felice 5 - 27100, Pavia (ITALY) \\ e-mail: emilio.zanettichini@unipv.it
}

March 2018

\begin{abstract}
The Generalized Smooth Transition Auto-Regression (GSTAR) parametrizes the joint asymmetry in the duration and length of cycles in macroeconomic time series by using particular generalizations of the logistic function. The symmetric smooth transition and linear auto-regressions are peculiar cases of the new parametrization. A test for the null hypothesis of dynamic symmetry is discussed. Two case studies indicate that dynamic asymmetry is a key feature of the U.S. economy. Our model beats its competitors in point forecasting, but this superiority becomes less evident in density forecasting and in uncertain forecasting environments.
\end{abstract}

Keywords: Density forecasts, Econometric modelling, Evaluating forecasts, Generalized logistic, Industrial production, Nonlinear time series, Point forecasts, Statistical tests, Unemployment.

JEL: C22, C51, C52 


\section{Introduction}

The U.S. business cycle is characterized by asymmetric fluctuations, as confirmed by a consolidated literature (Milas et al. (2006) and references therein). Defining asymmetry has been an important issue for many years. Sichel (1993) classifies two types of asymmetry: (i) the "steepness" that arises when contractions in the levels are steeper than expansions, corresponding to negative skewness in the first differences of the sample (or, in a graphical perspective, to asymmetry in the level axis); and (ii) the "deepness" that occurs when the series undergoes at an accelerating pace until a minimum, after which it starts to recover with quickly decreasing acceleration until it smoothly recovers the peak, corresponding to negative skewness in the levels (or asymmetry in time axis). Dynamic asymmetry occurs when these two definitions of asymmetry are combined. McQueen and Thorley (1993) use the term "sharpness" to refer to the probability that the transitions to and from the two regimes (expansion and contraction) are not identical. As a logical implication of these definitions, a dynamically asymmetric process can be identified by asymmetry in either conditional mean or in conditional density.

The primary focus of the present research is on out-of-sample forecasting for the U.S. index of industrial production (IIP) and unemployment rate (UN) ${ }^{1}$. These data are displayed in Figure 1 and a simple application of Sichel's test for deepness and steepness is reported in the first panel of Table 1. All of the series under consideration present at least some type of asymmetry: (i) the type of asymmetry in IIP changes, as quarterly and monthly growth rates amplify the steepness (the lower $p$-value is 0.07 for the sample at quarterly frequency and 0.06 for the sample in monthly frequency) with respect to the deepness (0.14 and 0.09, respectively), prevailing in the series in yearly growth rates (0.07 for quarterly sample and 0.05 in monthly sample). (ii) The UN is steep and deep, no matter the transform or frequency. A good

\footnotetext{
${ }^{1}$ All series, in both quarterly and monthly frequency, are in real time and can be downloaded by OECD - Main Economic Indicators.
} 
forecasting model for the U.S. business cycle must incorporate the dynamic asymmetry in all its possible types previously mentioned. To this aim, which is in most of this literature - particularly the first generation, such as Neftçi (1984); De Long and Summers (1984); Rothman (1991) - uses a piecewise linear autoregression with Markov-Switching mean or variance (MSAR) with a pre-specified number of unobserved states (usually two). This approach has been appreciated particularly for its easy implementation and close connections with algorithmic rules for dating; see Harding and Pagan (2006) for recent developments. The present research adopts an alternative strategy to treat the process as a continuum of observable states that oscillate between two extremes and to fit a general, flexible, nonlinear function over the observables using the smooth transition auto-regressions (STARs) introduced by Haggan and Ozaki (1981); Chan and Tong (1986) and developed by Teräsvirta (1994). These piecewise linear models are characterized by a nonlinear function of the transition variable, where a logistic transition is commonly postulated when the series is assumed to have asymmetric oscillations from its conditional mean. We argue that the use of this peculiar transition function is improper in many applications because the sigmoid of the logistic function is reflexively symmetric. As a consequence, the logistic STAR model can reproduce steepness but not deepness, while, as Clements and Krolzig (2003) explain, deepness is the only feature associated with asymmetry in the (un)conditional density of the process ${ }^{2}$.

The econometric literature provides two solutions: the first, proposed by Sollis et al. (1999) (SLN1) and Lundbergh and Teräsvirta (2006) (LT), is to exponentiate the STAR transition function; the second, suggested by Sollis et al. (2002) (SLN2) is to add a parameter inside the transition function in such a way as to control for the asymmetry of both of the transition function's tails by using a Heaviside indicator. Unfortunately, as Figure 2's panel (a) shows, neither of these solutions is free from idiosyncrasy: in the SLN2 model, the transition function can be non-smooth, while

\footnotetext{
${ }^{2}$ See Figure 1 of page 198 of the mentioned article.
} 
the SLN1 and LT parametrization conveys a smooth transition, but the effect of increasing the asymmetry parameter often translates to no more than a shift effect in the same transition function if it is not properly restricted. This shift could translate into an almost symmetric predictive density, as shown in Figures 8 and 10 in LT. In other words, the available models for time series limit the econometrician's ability to answer the question: 'When the series return to its original regime?' Here, our objective is to answer to another, more challenging question: 'Does the left tail of the process's (un)conditional distribution differ from its right tail and, if so, by how much?' This paper shows that the solution to this methodological question, which is interesting for its descriptive aims, improves the forecasting ability of the STAR models family.

In the next section, Section 2, we introduce a generalized version of the STAR model's logistic transition function with two parameters that govern the two tails of the logistic sigmoid and a logarithmic/exponential that can preserve the smoothness of the transition. The resulting Generalized STAR (GSTAR) model encloses the symmetric STAR - and, thus, linear AR - as special cases. An LM-type test for the null hypothesis that the two tails of the transition function are reflexively symmetric is discussed in Section 3. Section 4 illustrates the forecasting properties of the GSTAR model for the U.S. data, jointly with a discussion on the relevance of the empirical finding. Finally, Section 5 summarizes and concludes. The Supplement provides details on mathematical derivations and definitions, diagnostics, and simulations, as well as additional examples.

\section{Forecasting Models}

This section describes the non-linear and dynamic asymmetric models implemented in our analysis. For details on statistical inference, model specification, and parameter estimation, we refer readers to Teräsvirta et al. (2010), and to Canepa and 
Zanetti Chini (2016) for what concerns dynamic asymmetric specification. In what

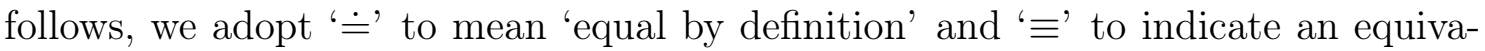
lence; bold is used for matrix notation; and $y_{t}$ is a realization of a (univariate) time series observed at $t=1-p, 1-(p-1), \ldots,-1,0,1, \ldots, T-1, T$. All estimated models for quarterly (monthly) samples include four (eight) lags, but all of the formulas written below refer to general autoregressive order $p$. Finally, all of the transition variables in our application are assumed to be lags of the dependent variable, so none of the models treated in this section requires exogenous variables.

\subsection{GSTAR Models}

The process $\left\{y_{t}\right\}_{t}^{T}$ follows a GSTAR(p) model if

$$
\begin{aligned}
y_{t} & =\boldsymbol{\phi}^{\prime} \mathbf{z}_{t}+\boldsymbol{\theta}^{\prime} \mathbf{z}_{t} G(\boldsymbol{\xi})+\epsilon_{t}, \quad \epsilon_{t} \sim \text { I.I.D. }\left(0, \sigma^{2}\right), \\
G(\boldsymbol{\xi}) & =\left(1+\exp \left\{-\prod_{k=1}^{K} h\left(c_{k}, s_{t}\right)\right\}\right)^{-1},
\end{aligned}
$$

where the $T \times 1$ vector $y_{t}$ is a dependent variable; $\mathbf{z}_{t}=\left(1, y_{t-1}, \ldots, y_{t-p}\right)^{\prime}, \boldsymbol{\phi}=$ $\left(\phi_{0}, \phi_{1}, \ldots, \phi_{p}\right)^{\prime}, \boldsymbol{\theta}=\left(\theta_{0}, \theta_{1}, \ldots, \theta_{p}\right)^{\prime}$ are autoregressive parameter vectors; $G(\boldsymbol{\xi}) \doteq$ $G\left(\boldsymbol{\gamma}, h\left(c_{k}, s_{t}\right)\right)$ is a transition function of the vector of nonlinear parameters $\boldsymbol{\xi}=$ $\left[\gamma, h\left(c_{k}, s_{t}\right)\right]$, which is formed by the vector $\gamma=\left(\gamma_{1}, \gamma_{2}\right)$ and a function of the $K$ location parameter $c_{k}$; the transition variable $s_{t}=y_{t-d}$, with $d>0$ denoting the delay, and defining $\eta_{t} \equiv\left(s_{t}-c\right)$ for ease of notation,

$$
h\left(\eta_{t}\right) \doteq \begin{cases}\gamma_{1}^{-1} \exp \left(\gamma_{1}\left|\eta_{t}\right|-1\right) & \text { if } \gamma_{1}>0 \\ 0 & \text { if } \gamma_{1}=0 \\ -\gamma_{1}^{-1} \log \left(1-\gamma_{1}\left|\eta_{t}\right|\right) & \text { if } \gamma_{1}<0\end{cases}
$$


for $\eta_{t} \geq 0(\mu>1 / 2)$ and

$$
h\left(\eta_{t}\right) \doteq \begin{cases}-\gamma_{2}^{-1} \exp \left(\gamma_{2}\left|\eta_{t}\right|-1\right) & \text { if } \gamma_{2}>0 \\ 0 & \text { if } \gamma_{2}=0 \\ \gamma_{2}^{-1} \log \left(1-\gamma_{2}\left|\eta_{t}\right|\right) & \text { if } \gamma_{2}<0\end{cases}
$$

for $\eta_{t}<0(\mu<1 / 2)$. The transition function $G(\cdot, \cdot)$ is continuous in $\gamma$. Equation (3) (equation (4)) models the higher (lower) tail of the transition function, so it allows for the asymmetric behavior introduced by the slope parameter $\gamma_{1}\left(\gamma_{2}\right)$, which controls the velocity of the transition in each half of the same function. When $\gamma_{1}, \gamma_{2}>0\left(\gamma_{1}, \gamma_{2}<0\right), h\left(\eta_{t}\right)$ is an exponential (logarithmic) rescaling that increases more quickly (more slowly) than a standard logistic function does. This parametrization ensures that the global slope remains unchanged with respect to the traditional symmetric logistic function. The requirement that $h(\cdot)=0$ when the slope parameter is zero is necessary for ease of exposition and allows us to build a test for the null of linearity against of dynamic asymmetry, thereby to nest the results in previous literature. The associated Supplement discusses the original, slightly different definition of generalized logistic function introduced by Stukel (1988), according to which $h(\cdot)=\eta_{t}$ when the slope is zero. This definition is adopted in next Section 3 for testing. Moreover, in our applications, we assume $K=1$, although other distributions of exponential family can be achieved easily ${ }^{3}$.

The Generalized Logistic function is plotted in Figure 2's panel (b). If we interpret the support of this function as a probability of recession, the resulting sigmoid is consistent with the Sichel (1993) definition of dynamic asymmetry, where, according to the values of the two slope parameters, the observables are associated with an

\footnotetext{
${ }^{3}$ The Generalized Logistic is the basis for other transition functions, such as the (Generalized) Double Logistic, which allows the sharpness to be incorporated into a STAR model; see the Supplement. Another other important case is $K=2$, corresponding to the generalized exponential STAR (GESTAR), where parameters $\boldsymbol{\phi}+\boldsymbol{\theta} G\left(\gamma, \boldsymbol{c}, s_{t}\right)$ change asymmetrically around the mid-point $\left(c_{1}+c_{2}\right) / 2$ and where the generalized logistic function attains its minimum, $\min _{G} G(\cdot) \in[0,1 / 2]$. However, this specification is not used in our application and is not discussed; see Canepa and Zanetti Chini (2016) and the Supplement for details.
} 
abrupt (smooth) acceleration in the first half and to a smooth (abrupt) deceleration in the second half. Deepness occurs when acceleration is abrupt in the first half and moderate in second half; on the opposite side, steepness occurs when the acceleration in the first half is moderate and abrupt in the second half. The lack of any identification restrictions in the two slope parameters guarantees that the two types of asymmetry can coexist.

The one-to-one relationship between the new dynamically asymmetric model and the unconditional density of the nonlinear part of the process is shown in Figure 3's first and second rows. Nonlinear models are always associated with a bimodal distribution; more specifically, in GSTAR parametrization, each $\gamma$ governs one of these modes. Three special cases are (i) $h\left(\eta_{t}\right)=\eta_{t}$, which suggests that the function nests a one-parameter symmetric logistic STAR model with slope $\gamma_{1}=\gamma_{2}=\gamma$; as shown in Figure 3's fourth row, a value of $\gamma=1$ with 1,000 observations is sufficient to see that density tends to concentrate in the two extremes. (ii) When $\gamma \rightarrow+\infty$, $G(\cdot, \cdot)$ nests an indicator function $I_{\left(s_{t}>c\right)}$; in this case (not shown) the distribution of the process degenerates a rectangle-' $U$ '. (iii) When $\gamma=0, G(\cdot, \cdot)$ nests a straight line around $1 / 2$ for each transition variable $s_{t}$ and the distribution becomes symmetric (Figure 3's third row). Each of these three special cases is described in this section. An important assumption is that $Q(z)=z^{p}-\phi_{1} z^{p-1}-\cdots-\phi_{p}=0$ has its roots inside the unit circle if the process is characterized by $G\left(\mathbf{0}, h\left(\eta_{t}\right)\right)$, which suggests that the model is stationary and ergodic under the null hypothesis of linearity ${ }^{4}$. Finally, $\left\{\epsilon_{t}\right\}_{t}^{T}$ is assumed to be a martingale difference sequence with respect to the history of the time series up to time $t-1$, denoted as $\Omega_{t-1}=\left[y_{t-1}, \ldots, y_{t-p}\right]$, i.e., $E\left[\epsilon_{t} \mid \Omega_{t-1}\right]=0$. This assumption is sufficient to build tests based on artificial regressions, as demonstrated in Davidson and McKinnon (1990), so an important consequence of this assumption is that the test discussed in Section 3 and the three diagnostic tests discussed in the Supplement can still be meaningful if the normality

\footnotetext{
${ }^{4}$ This assumption can be relaxed, as in Kapetanios et al. (2003); Vougas (2006).
} 
hypothesis is rejected.

\section{$2.2 \quad$ STAR Models}

When $\gamma_{1}=\gamma_{2}$ in (1)-(4), the GSTAR model nests a traditional (possibly, Multiple Regime) STAR model (MRSTAR, henceforth):

$$
y_{t}=\boldsymbol{\phi}^{\prime} \mathbf{z}_{\mathbf{t}}+\boldsymbol{\theta}^{\prime} \mathbf{z}_{\mathbf{t}} \sum_{m=1}^{M} G\left(\boldsymbol{\gamma}, \mathbf{c}, s_{t}\right)+\epsilon_{t}, \quad \epsilon_{t} \sim i . i . d .\left(0, \sigma^{2}\right)
$$

where $y_{t}, \mathbf{z}_{\mathbf{t}}, \boldsymbol{\phi}, \boldsymbol{\theta}$ have been defined previously. The transition function $G\left(\boldsymbol{\gamma}, \boldsymbol{c}, s_{t}\right)$ is a continuous function in the transition variable $s_{t}$, where the parameter vector $\gamma=\left(\gamma_{1}, \ldots, \gamma_{m}, \ldots \gamma_{M}\right)$ controls the velocity of the $M$ transitions, with $\mathbf{c}=$ $\left(c_{1}, \ldots, c_{m}, \ldots c_{M}\right)$ assumed to be a vector of transition parameters

When $K=1$, equations (6) and (5) define the first-order (Multiple-Regime) Logistic STAR (MRLSTAR) model,

$$
\begin{array}{r}
G\left(\boldsymbol{\gamma}, \boldsymbol{c}, s_{t}\right)=\left(1+\exp \left\{-\gamma_{M} \prod_{k=1}^{K}\left(s_{t}-c_{m}\right)\right\}\right)^{-1}, \\
\gamma_{1}>0, \ldots, \gamma_{m}>0, \ldots, \gamma_{M}>0, \quad c_{1}<\cdots<c_{m}<\cdots<c_{M}
\end{array}
$$

where conditions on $\gamma$ and $c$ in equation (6) are identifying restrictions, making the symmetric (MR)STAR fundamentally different from GSTAR in (1), where no additional identification restriction is needed. Under the logistic specification, $\boldsymbol{\phi}+\boldsymbol{\theta} G\left(\gamma, \boldsymbol{c}, s_{t}\right)$ changes monotonically from $\boldsymbol{\phi}$ to $\boldsymbol{\phi}+\boldsymbol{\theta}$ as a function of $s_{t}$. For applications of this model in the U.S. business cycle, Anderson and Teräsvirta (1992); Rothman (1998), and others. 


\section{$2.3 \quad$ (SE)TAR Models}

When $\gamma_{1}=\gamma_{2} \rightarrow \infty$ in (1)-(4) (or, equivalently $\gamma_{m} \rightarrow \infty$ the model (5), the (G)STAR nests a SETAR model (Tong, 1983):

$$
y_{t}=\sum_{j=1}^{r+1}\left(\phi_{j}^{\prime} \mathbf{z}_{\mathbf{t}}\right) I\left(y_{t-d} \leq c_{j}\right)+\sum_{j=1}^{r+1}\left(\phi_{j}^{\prime} \mathbf{z}_{\mathbf{t}}\right) I\left(y_{t-d}>c_{j}\right)+\epsilon_{j t} \quad \epsilon_{j t} \sim i . i . d .\left(0, \sigma_{j}^{2}\right)
$$

where $\phi$ and $\mathbf{z}_{\mathbf{t}}$ are defined as in previous models, $s_{t}$ is a continuous switching random variable, $c_{0}, c_{1}, \ldots, c_{r+1}$ are threshold parameters, $c_{0}=-\infty, c_{r+1}=+\infty$,

$j=1, \ldots, r$. The multiple regime hypothesis is investigated via the LM test, and the most likely number of regimes is obtained by iteration. When $\gamma_{1}=\gamma_{2}=0$ in model (1)-(4) - or, equivalently, $\gamma=0$ and $M=1$ in model (5) - the transition function is $G\left(\gamma_{m}, \boldsymbol{c}, s_{t}\right) \equiv 1 / 2$ and the equation collapses into a linear autoregression; see Figure 2's panel (b) .

\section{Testing for Dynamic Symmetry}

According to the definition of GSTAR model given in Section 2, the dynamic asymmetry of the series is modelled by parameters $\gamma_{1}$ and $\gamma_{2}$. Hence, a test for the presence of dynamic asymmetry in the process $y_{t}$ requires the following hypothesis system:

$$
\begin{aligned}
& H_{0}: \gamma_{1}=0 \text { and } \gamma_{1}=0 \text { in }(3)--(4), \\
& H_{1}: \gamma_{1} \neq 0 \text { and } \gamma_{1} \neq 0 \text { in }(3)--(4) .
\end{aligned}
$$

Testing for the null hypothesis of dynamic symmetry requires substituting $h\left(\eta_{t}\right)=\eta_{t}$ in models (3)-(4), as otherwise the null hypothesis becomes linear. In both cases, the alternative hypothesis remains dynamic asymmetry. The idea of this test is the same as that of Luukkonen et al. (1988): the model is linearized via Taylor expansion in order to build an artificial regression whose coefficients incorporate these two 
slopes. Since Luukkonen et al. assume just one slope parameter, their notation must be modified. Consider (1) with $\left.G\left(\boldsymbol{\gamma}, h\left(\eta_{t}\right)\right)\right|_{\boldsymbol{\gamma}=\mathbf{0}}$ and define $\boldsymbol{\tau}=\left(\boldsymbol{\tau}_{\mathbf{1}}, \boldsymbol{\tau}_{\mathbf{2}}\right)^{\prime}$, where $\boldsymbol{\tau}_{\mathbf{1}}=\left(\phi_{0}, \boldsymbol{\phi}^{\prime}\right)^{\prime}, \boldsymbol{\tau}_{\mathbf{2}}=\boldsymbol{\gamma}$. Let $\hat{\boldsymbol{\tau}}_{\mathbf{1}}$ the LS estimator of $\boldsymbol{\tau}_{\mathbf{1}}$ under $H_{0}: \boldsymbol{\gamma}=\mathbf{0}, \hat{\boldsymbol{\tau}}=\left(\boldsymbol{\tau}_{\mathbf{1}}^{\prime}, \mathbf{0}^{\prime}\right)^{\prime}$. Let $\mathbf{z}_{\mathbf{t}}(\boldsymbol{\tau})=\frac{\partial \epsilon_{t}}{\partial \boldsymbol{\tau}}$ and $\hat{\mathbf{z}}_{\mathbf{t}}=\mathbf{z}_{\mathbf{t}}(\hat{\boldsymbol{\tau}})=\left(\hat{\mathbf{z}}_{\mathbf{1}, \mathbf{t}}, \hat{\mathbf{z}}_{\mathbf{2}, \mathbf{t}}\right)$, where the partition conforms to that of $\boldsymbol{\tau}$. Then the general form of the LM statistic is:

$$
S(\boldsymbol{\Xi})^{L M}=\frac{1}{\hat{\sigma}^{2}} \hat{\mathbf{u}}^{\prime} \hat{\mathbf{Z}}_{\mathbf{2}}\left(\hat{\mathbf{Z}}_{\mathbf{2}}^{\prime} \hat{\mathbf{Z}}_{\mathbf{2}}-\hat{\mathbf{Z}}_{\mathbf{2}}^{\prime} \hat{\mathbf{Z}}_{\mathbf{1}}\left(\hat{\mathbf{Z}}_{\mathbf{1}}^{\prime} \hat{\mathbf{Z}}_{\mathbf{1}}\right)^{-1} \hat{\mathbf{Z}}_{\mathbf{1}}^{\prime} \hat{\mathbf{Z}}_{\mathbf{2}}\right)^{-1} \hat{\mathbf{Z}}_{\mathbf{2}}^{\prime} \hat{\mathbf{u}}
$$

where $\hat{\mathbf{u}}$ is previously defined, $\hat{\sigma}^{2}=\frac{1}{T} \sum_{1}^{T} \hat{u}_{t}^{2}$ and $\hat{u}_{t}=y_{t}-\hat{\boldsymbol{\tau}}_{1}^{\prime} \mathbf{z}_{\mathbf{t}}, \hat{\mathbf{Z}}_{\mathbf{i}}=\left(\hat{\mathbf{z}}_{\mathbf{i} \mathbf{1}}, \ldots, \hat{\mathbf{z}}_{\mathbf{i t}}, \ldots, \hat{\mathbf{z}}_{\mathbf{i T}}\right)^{\prime}$, $i=\{1,2\}, t=1, \ldots, T$. When the model is a GLSTAR, $\hat{\mathbf{z}}_{\mathbf{1}, \mathbf{t}}=-\mathbf{z}_{\mathbf{t}}=-\left(1, y_{t-1}, \ldots, y_{t-p}\right)^{\prime}$ while $\left.\hat{\mathbf{z}}_{\mathbf{2} \mathbf{t}} \equiv \frac{\partial^{2} u_{t}}{\partial \boldsymbol{\gamma} \partial \boldsymbol{\gamma}^{\prime}}\right|_{\boldsymbol{\gamma}=\mathbf{0}}=-\frac{1}{2}\left\{\theta_{20}\left[y_{t}\left(y_{t-d}\right)\right]-c y_{t} \boldsymbol{\theta}^{\prime} \mathbf{z}_{\mathbf{t}}+\boldsymbol{\theta}_{\mathbf{2}}^{\prime} \mathbf{z}_{\mathbf{t}} y_{t} y_{t-d}\right\}$, where $d$ is the delay parameter. As for symmetric case, the nuisance parameters $\theta_{0}$ and $\overline{\boldsymbol{\theta}}=\left[\theta_{1}, \ldots, \theta_{p}\right]^{\prime}$ are not identified under the null hypothesis; see Davies (1977). In our framework, the linearization of GLSTAR model leads to the following auxiliary regression:

$$
\hat{u}_{t}=\hat{\mathbf{z}}_{\mathbf{1} \mathbf{t}}^{\prime} \tilde{\boldsymbol{\beta}}_{\mathbf{1}}+\sum_{j=1}^{p} \beta_{2 j} y_{t-j} y_{t-d}+\sum_{j=1}^{p} \beta_{3 j} y_{t-j} y_{t-d}^{2}+\sum_{j=1}^{p} \beta_{4 j} y_{t-j} y_{t-d}^{3}+v_{t},
$$

where $v_{t}$ is a N.I.D. $\left(0, \sigma^{2}\right)$ process, $\tilde{\boldsymbol{\beta}}_{\mathbf{1}}=\left(\beta_{10}, \boldsymbol{\beta}_{\mathbf{1}}^{\prime}\right)^{\prime}, \beta_{10}=\phi_{0}-(c / 4) \theta_{0}, \boldsymbol{\beta}_{\mathbf{1}}=$ $\boldsymbol{\phi}-(c / 4) \boldsymbol{\theta}+(1 / 4) \theta_{0} \mathbf{e}_{d}, \mathbf{e}_{d}=(0,0, \ldots, 0,1,0, \ldots, 0)^{\prime}$ with the $d$-th element equal to unit and $T_{3}(G)=f_{1} G+f_{3} G^{3}$ is the third-order Taylor expansion of $G(\boldsymbol{\Xi})$ at $\boldsymbol{\gamma}=\mathbf{0}, f_{1}=\partial G(\boldsymbol{\Xi}) /\left.\partial \boldsymbol{\Xi}\right|_{\boldsymbol{\gamma}=\mathbf{0}}$ and $f_{3}=(1 / 6) \partial^{3} G(\boldsymbol{\Xi}) /\left.\partial \boldsymbol{\Xi}\right|_{\boldsymbol{\gamma}=\mathbf{0}}, G(\boldsymbol{\Xi})$ being defined in previous section ${ }^{5}$. The null hypothesis is

$$
H_{0}^{\prime}: \beta_{2 j}=\beta_{3 j}=\beta_{4 j}=0 j=1, \ldots, p,
$$

The test statistic:

$$
L M_{1}=\left(S S R_{0}-S S R\right) / \hat{\sigma}_{v}^{2},
$$

\footnotetext{
${ }^{5}$ Notice the difference from similar expressions in Teräsvirta (1994): here $\boldsymbol{\tau}_{\mathbf{2}}$ is a vector and $\hat{\mathbf{z}}_{\mathbf{2 t}}$ is the double (it was $-\frac{1}{4}\{\cdots\}$ ). The LM statistic and the terms in the auxiliary regression remain unchanged.
} 
with $\mathrm{SSR}_{0}$ and SSR denoting the sum of the squared estimated residuals from the estimated auxiliary regression (11) and under the null and alternative, respectively, and $\sigma_{v}^{2}=(1 / T) S S R$ has an asymptotic $\chi_{3 p}^{2}$ distribution under $H_{0}^{\prime}$. A similar ar-

gument with different definitions of $\hat{u}_{t}, \tilde{\boldsymbol{\beta}_{\mathbf{1}}}, \boldsymbol{\beta}_{\mathbf{1}}, H_{0}^{\prime}$, holds for the exponential and double exponential cases. Alternatively, a Score test for the null hypothesis of dynamic asymmetry can be implemented via standard inference; see Stukel (1988) and the Supplement. Our experience suggests the empirical power of Taylor expansionbased tests is almost always higher than that of Stukel's test.

\section{Illustrations}

\subsection{Set-up}

In this section the GSTAR model is applied to the U.S. data introduced in Section 1. The peculiar logarithmic/exponential rescaling property of the generalized logistic transition function makes our parametrization particularly useful in fitting the variables characterized by a high level of regime persistence. Therefore, we estimate eight models in order to control for the possible dominance of deepness against sharpness: for each variable (IIP and UN) we consider samples in quarterly and monthly frequencies, using either yearly or monthly/quarterly growth rates. The literature on point forecasting and evaluation of individual density forecasts under linear models is well established; see Timmermann (2006) and Corradi and Swanson (2006). To evaluate the forecasts, we adopt four measures of point forecast accuracy: the mean forecast error (MFE), the symmetric mean absolute percentage error (sMAPE), the median relative absolute error (mRAE), and the root mean square forecast error (RMSFE). For accuracy in the density forecasts, we use the logarithmic score (LogS), the quadratic score (QSR), the continuous-ranked proba- 
bility score (CRPS), and the quantile score (qS $)^{6}$. When the model is nonlinear and its nonlinear function is known, the one-step forecast is immediately available by the least-square criterion. The multi-step ahead forecast is not available in closed form, so it requires numerical integration. Hence, at $t+1$, we generate $1, \ldots, M$ draws - for example, from model (1)-(4) conditionally on the estimated nonlinear parameters $\boldsymbol{\xi}$ - and obtain the forecast $y_{t+1} \sim f\left(y_{t+1}+\epsilon_{t+1}^{(m)} ; \boldsymbol{\xi} \mid I_{t}\right)$. This forecast is collected to draw, at $t+2$, the forecast $y_{t+2} \sim f\left(y_{t+2}+\epsilon_{t+2}^{(m)} ; \boldsymbol{\xi} \mid I_{t}, y_{t+1}^{(m)}\right)$, and so on until, at $t+h$, the forecast $y_{t+h \mid t}=f\left(y_{t+h}+\epsilon_{t+h} \mid I_{t}, y_{t+1}^{(m)}, \ldots, y_{t+h-1}^{(m)}\right)$ is obtained and then evaluated as:

$$
y_{t+h}^{M C}=\frac{1}{M} \sum_{m=1}^{M} y_{t+h \mid t}^{(m)} .
$$

The Monte-Carlo approach requires making assumptions on the distribution of random numbers $\epsilon_{t}$, which, as we will see in this section, might influence density forecasts. This problem can be partially avoided by block-bootstrapping the sample: the series is divided into blocks of magnitude $b>1$, which are then sampled with replacement, and this is done for every possible contiguous element in the original sample. Thus, the sampled blocks are attached, obtaining the new bootstrap series $\left(\tilde{y}_{t}^{(1)}, \ldots, \tilde{y}_{t}^{(i)}, \ldots, \tilde{y}_{t}^{(B)}\right)$ from the same model. Finally, we compute the $M_{B}^{b}$ forecasts for $\tilde{y}_{t+1}, \tilde{y}_{t+2}, \ldots, \tilde{y}_{t+h}$ as before, to arrive at $\tilde{y}_{t+h}=g\left(\tilde{y}_{t+h}+\tilde{\epsilon}_{t+h}^{(i)} \mid I_{t+h-1}\right)$ and:

$$
\tilde{y}_{t+h}^{B}=\frac{1}{M_{B}^{b}} \sum_{B=1}^{M_{B}^{b}} \tilde{y}_{t+h \mid t}^{(B)} .
$$

In our application we adopt a moving block-bootstrap algorithm with $b=10$ and $B=10,000$ draws, which allows us to avoid making assumptions about the distribution of estimated residuals and gives us a forecast that is robust to model parameter uncertainty; see Efron and Tibshirani (1993), Chapter 8. Then we check to see whether our model performs better than its linear and symmetric competitor(s) us-

\footnotetext{
${ }^{6}$ The choice of these measures of predictive performances was made for ease of treatment and ease of comparison in the literature and implies no preference.
} 
ing the Diebold and Mariano (1995) test ${ }^{7}$, the Giacomini and White (2006) test, and the Amisano and Giacomini (2007) test for equal predictive ability for couples of forecasts. Under the null hypothesis, there is no evidence of that the GSTAR or its linear or symmetric equivalent is superior.

\subsection{The U.S. Industrial Production}

According to the $p$-values reported in Table 1, the null hypotheses of linearity and dynamic symmetry are always rejected if the significance threshold is at 0.10 . Nevertheless, detecting nonlinearity and dynamic asymmetry is more difficult when quarterly and monthly growth rates are considered. For example, in the quarterly series in quarterly growth rates, the $p$-values are higher $(0.07$ and 0.04$)$ than those that correspond to the series in yearly growth rates (which $p$-value is 0.0037 for the null of linearity and 0.0005 for the null of symmetry). Monthly data samples confirm this weak asymmetry. None of the estimated models requires the use of a second transition function to capture additive dynamic asymmetry or evidence of time-varying parameters. The $p$-values of diagnostic tests for the null of serial uncorrelation are often lower than 0.05. Nevertheless, the effects in terms of $p$-value are limited, although only by increasing the autoregressive order considerably. Thus, if the other diagnostic tests are passed, the choice of an order $p=4$ appears merely to preserve parsimony.

In three series (both quarterly samples and the monthly sample in yearly growth rates), the estimated slopes have opposite signs ( $\gamma_{1}$ negative and $\gamma_{2}$ positive) and are, in most cases, statistically significant, with the magnitude higher in $\gamma_{1}$. On the other hand, in the series of monthly growth rates, both estimated slopes are positive, and the $p$-values of linearity and asymmetry tests are higher than other

\footnotetext{
${ }^{7}$ Since the $\operatorname{AR}(\mathrm{p})$ and $\operatorname{STAR}(\mathrm{p})$ models are nested in the $\operatorname{GSTAR}(\mathrm{p})$ specification proposed here, the inference of this test is severely biased, as shown in West (1996), so equivalent tests, such as that in Clark and McCracken (2001), should be employed. Nevertheless, we use the classical Diebold-Mariano for a preliminary check. As our illustrations will show, the resulting $p$-values are often counterintuitive. In any case, the $p$-values of the Clark-McCracken test do not change our conclusions, so they are omitted for reasons of space.
} 
samples. Figures 4 and 5 plot the time path of GSTAR's and symmetric MRSTAR's estimated transition functions for samples in quarterly and monthly frequency, respectively. The sigmoid associated with each transition function in Figures 4 and 5 can be interpreted as a global indicator of the series' reactiveness in the contraction phase (that is, 0 is the extreme of contraction and 1 is the maximum of expansion). The deepness is dominant in the sigmoids that correspond to the series at quarterly frequency in yearly growth rates, while the symmetric model behaves as a step function with almost all observations taking the value of 1 and some 0 , so it is similar to a SETAR model.

On the other side, the MRSTAR sigmoid that corresponds to quarterly growth rates is almost linear, while the GSTAR sigmoid is steep and deep. In monthly frequency data, deepness is still great when yearly growth rates are considered, while monthly growth rates are characterized by high steepness and moderate deepness. In both of the monthly frequency series, the MRSTAR transition function is still a zero-onetype, with the only exception in the first function in monthly growth rates. Hence, the symmetric models fail to capture dynamic asymmetry. These results seem compatible with Sichel's conclusion that deepness prevails in the production sector, but according to our model, such a dominance of deepness is sensitive to the basis of the growth rates and never annihilates the role of steepness.

The predictive performances of the GSTAR, MRSTAR, and linear AR models are reported in Tables 2 and 3 for quarterly and monthly data, respectively. Our parametrization is preferable to both alternatives for three measures of point forecasting and two density measures of the total of four measures considered. LogS, the linear specification, is preferred for short-term forecasts and the nonlinear symmetric model is preferred for medium-term forecasts (2 and 4 quarters ahead). On the other hand, in monthly data, the GSTAR model is preferred in three point measures of four and just one density measure (the $\operatorname{LogS}$ ) of four, while the quadratic and quantile scores support the symmetric nonlinear parametrization, so the linear 
model is preferable under CRPS. Tables 4 and 5 report the predictive accuracy measures for the same experiment, where forecasts of models for quarterly and monthly data samples are obtained by the block-bootstrap algorithm.

Parameter uncertainty in estimation reduces the point predictive performances of asymmetric models. The symmetric MRSTAR performs better than the GSTAR in one measure (mRAE), and the linear autoregression is preferred in the majority of horizons in MFE and RMFE. On the other side, the asymmetric specification overperforms in three density measures of four - that is, one more than the experiment with no uncertainty. In monthly samples, the GSTAR model is preferable in almost all leads of MFE and mRAE, and in the others the symmetric model prevails in other point measures - although in density measures, only under QS.

The hypothesis of no equal predictive ability is investigated in Table 6 for models of quarterly samples and in Table 7 for models of monthly data samples. The DieboldMariano test does not reject the null hypothesis of no improvement in forecasting ability for nonlinear and dynamically asymmetric specifications with respect to linear (and symmetric) ones. This result appears counterintuitive, since the linearity and asymmetry tests suggest the opposite. Hence, given that the Diabold-Mariano statistics has been built for non-nested models, we use them as an additional check. The more general Giacomini-White test allows for improvements in the forecasting ability of GSTAR model in short-run horizons, although the $p$-values blow up as horizons increase, so the evidence of an improvement decays rapidly in the long run. The Amisano-Giacomini test supports the GSTAR model only under CRPS; however, if the choice is between a nonlinear symmetric model and linear speciPcation, the former is also supported by the quantile measure. In monthly samples the gain in the forecasting ability of asymmetric models is considerably greater for both asymmetric and nonlinear symmetric specifications; the Amisano-Giacomini test supports nonlinear specification and restricts the preference for the GSTAR model to LogS. 


\subsection{The U.S. Unemployment Rate}

A graphic inspection of the series of UN in Figure 1 is sufficient to reveal that this variable is strongly countercyclical and, with respect to the industrial production, its business cycle phases are often exacerbated. This finding is confirmed by the tests for linearity and dynamic symmetry in Table 1: the series in monthly growth rates (the $p$-value of which is 0.04 ) is near the limit and is high if the high number of observations is considered ${ }^{8}$. All of the models pass the diagnostic tests for the null of no additive asymmetry and parameter constancy. The hypothesis of serial uncorrelation is rejected only in quarterly samples. Still, we maintain four lags in the model specification because there is no evidence of satisfactory improvement in p-values in tests for serial uncorrelation unless the autoregressive order is augmented up to $8^{9}$. With the counter-cyclical nature of this variable, the estimated slopes are opposite in sign with respect to industrial production in all the series: $\gamma_{1}$ is positive and $\gamma_{2}$ is negative with higher magnitude in $\gamma_{1}$. The only exception is represented by quarterly series in yearly growth rates, where the parameters become 1 and -1 , respectively.

Figures 5 and 6 depict the transitions of the (G)STAR models for samples at quarterly and monthly frequency, respectively. The sigmoids of UN are characterized by high steepness and moderate deepness, thus confirming Sichel's conclusion that Okun's law is dynamically asymmetric. Moreover, we find that the GSTAR model allows us to parametrize the natural rate of unemployment, which is shown in the lower tail of G: in three cases of four, the sigmoid does not begin at 0 but at 0.1 in the

\footnotetext{
${ }^{8}$ However, in a previous version of this paper, we considered also the null hypothesis that the model is a GSTAR with different slopes, a hypothesis that strictly follows Stukel's original methodology. This consideration changes all of the results, which then indicate nonlinear asymmetric behavior only in the samples of yearly growth rates. Evidence from a Monte Carlo study of these more restrictive tests lead us to discourage their use because of their considerable conservatism. See the Supplement for additional details.

${ }^{9}$ In previous versions of this paper, the unemployment rate was analyzed only in monthly frequency. We set $p=4$, and an error in code in the estimation step led us to reconsider the whole modelling procedure and the diagnostic test. We thank an anonymous referee for observing this point.
} 
series of yearly growth rates, and at 0.3 in the series of quarterly/monthly growth rates. This important macroeconomic feature is impossible to capture using symmetric models, where the sigmoid always begins at 0 . According to the comparison of predictive accuracy reported in Table 2, the GSTAR parametrization allows for a significant forecasting gain with respect to both AR and MRSTAR models in three measures of point forecasting and almost every density measure. Such supremacy of the dynamically asymmetric specification is confirmed by monthly series (Table 3) for point measures, with some exceptions for short-run and very long-run leads. On the opposite side, in density measures the better performance of GSTAR (in long-run horizons) is an exception: while the QS supports the symmetric nonlinear parametrization, in CRPS and qS the linear model is preferred. Parameter uncertainty annihilates all predictive performances of asymmetric models. As shown in Table 4, the MRSTAR beats GSTAR in two point measures (MFE and mRAE) and two density measures (QS and qS), and the linear autoregression wins in other cases. Between the models for monthly samples (Table 5), the GSTAR is the best performer for almost two leads of MFE and almost all leads of mRAE and in the quadratic measure for density.

The Giacomini-White test allows for improvements in the MRSTAR model's forecasting ability against the linear model in three horizons of four in quarterly data (Table 6); the GSTAR model overperforms in short-run and medium-run horizons, but when the horizons increase, the $p$-values blow up and the evidence of forecasting improvement decays rapidly. The results of the Amisano-Giacomini test are equivalent to the results for industrial production: the GSTAR model beats MRSTAR only under CRPS, while the MRSTAR beats AR in CRPS and qS. In models of monthly data (Table 7), both the Diebold Mariano test and the Giacomini-White test reject the null hypothesis considerably more strongly than they do in models of quarterly data; the Amisano-Giacomini test allows for improvements in forecasting ability for the case of MRSTAR models against linear models, but only under QS. In 
the same test, GSTAR performances are also better than its competitors in long-run horizons of LogS, qS and CRPS, while in short-run forecasts, the MRSTAR remains the benchmark in almost all measures.

\subsection{Discussion}

What do we learn from this empirical investigation? First, the GSTAR model characterizes the dynamic asymmetry of the U.S. business cycle more accurately than its nonlinear competitor does because cyclical movements in the data and their phases are reproduced considerably more precisely than they are in the traditional parametrization. This result is confirmed by the transition functions' time paths, which are consistent with NBER recession dates in most of the cases. Since the literature is almost completely focused on Markov-Switching and Threshold autoregressions (see, e.g., Engel et al. (2005); Chauvet and Piger (2008)), the effect of the new model on dating algorithms remains unknown. Despite this difference in methodology, our results support McQueen and Thorley's main conclusion that theoretical macroeconomic models should not treat shocks in production and unemployment at the end of a recession as mirror images of the cutbacks at the end of a phase of expansion.

Second, such superior descriptive accuracy is usually associated with an improvement in point forecasting ability. Ferrara et al. (2015) investigate the forecasting properties of a large set of models - they also add MSARs and time-varying ARs after the Great Recession using a dataset that is larger than ours. Their conclusions are that (i) the predictive gain that arises from non-linear models is not systematic and, when it does exist, is small ${ }^{10}$. This evidence is explained by Stock and Watson (2012) hypothesis that the Great Recession can be seen as a sequence of unusually

\footnotetext{
10"Indeed, the results are rather mixed and depend strongly on the evaluation period. However, predictive gains that stem from nonlinear models may arise from variables that experienced clear regime changes over the sample, such as interest rates, for instance. When comparing the performances of non-linear models, the TVAR model seems to be very similar to the AR model [...] and the MSAR model often leads to the poorest results. On the other hand, the TAR and LSTAR models occasionally perform quite well' (Ferrara et al., 2015, page 678).
} 
large shocks. (ii) They assert that using exogenous variables in time series models is globally preferable with respect to auto-regressions. Although, for reasons of space, we do not consider models with exogenous variables, our evidence supports Ferrara et al.'s first conclusion about dynamically asymmetric models' low gain in terms of forecasting performance only when considering density measures. We confirm Kascha and Ravazzolo (2010) evidence for the relationship between highest LogS and lower RMSFE as not one-to-one but as commonly (and implicitly) postulated ${ }^{11}$. Unlike Ferrara et al., we observe that the Great Recession provides an important motivation for the use of dynamic asymmetric models and, in general, for the use of a smooth-transition autoregressive family. In fact, controlling for the inclusion of data after 2007 leads to an important increase in test for dynamic asymmetry' $p$-value ${ }^{12}$. However, our forecasting exercise assumes $E\left[\epsilon_{t}^{2} \mid \Omega_{t-1}\right]=\sigma^{2}$ - that is, that the conditional variance of the process $\left\{\epsilon_{t}\right\}_{t}^{T}$ is constant. Recent advancements in this strand of literature allow this restriction to be relaxed in STAR models; see González-Rivera (1998); Lundbergh et al. (2003); McAleer and Medeiros (2008); Amado and Teräsvirta (2013); Silvennoinen and Teräsvirta (2013). According to Clark and Ravazzolo (2015), incorporating stochastic (time-varying) volatility in simple macroeconometric models improves their forecasting properties substantially. We emphasize that the role of the dynamic asymmetry, particularly its intersection with stochastic/time-varying volatility, has never been investigated. Understanding the role of parameter uncertainty is not easy, and it has limited influence on the forecasting ability of models for pro-cyclical variables. On the other hand, it downgrades most dynamic asymmetric models of anti-cyclical proxies. Therefore, STARs' returns are often preferable when this feature must be taken into consideration. Finally, our forecast comparisons are based on statistical tests that are originally developed for linear models. Little is known about the impact on uncertain environ-

\footnotetext{
${ }^{11}$ We emphasize that this result is confirmed by our additional examples in the Supplement, where other (not only economic) datasets have been analyzed.

${ }^{12}$ We do not show the results because of space limits. Additional results can be provided on request.
} 
ments if dynamic asymmetry is assumed. The Giacomini and Rossi (2010)'s fluctuation test was supposed to clarify our findings, but according to our preliminary results (not shown), the fluctuation test seems to over-reject the null hypothesis, as they do also when linear autoregression is a reasonable hypothesis. Therefore, we postpone this important issue for later methodological investigation.

\section{Conclusions}

The generalized logistic function is applied to the STAR models family as a simple, statistically feasible way to capture the dynamic asymmetry in the conditional mean of a time series. Because of its logarithmic (exponential) rescaling, the resulting GSTAR model ensures the smoothness of the transition function by construction without requiring additional effort to determine what concerns identification and estimation and allows us to model the two (or, possibly more, if multiple transition functions are required) modes in the process's density function. An application of the new model to two proxies of the U.S. business cycle, each one in four transformations, allows us to support most of the previous literature's findings and to improve the quality of the econometric modelling. A properly specified GSTAR model for industrial production and the unemployment rate leads to a substantial gain in point forecasting ability. According to our parametrization, dynamic asymmetry affects the process's conditional mean almost exclusively. Its detection in higher conditional moments and its statistical treatment in unstable forecasting environments remain unexplored. Moreover, the univariate analysis provided here limits our empirical results' relevance in terms of policy implications. Since the GSTAR model's high level of flexibility makes it a valid tool with which to model and forecast other prominent, nonlinearly behaving economic and financial variables, such as monetary aggregates and risk measures, we encourage further research efforts in this direction. 


\section{Acknowledgements}

This paper, originally titled "Generalizing Smooth Transition Autoregressions" was developed when the author was a visiting Ph.D. student at the Center for Research in Econometric Analysis of Time Series (DNRF78) and was funded by the Danish National Research Foundation. The author gratefully acknowledges the hospitality and the stimulating research environment provided by Niels Haldrup, the supportive and helpful supervision of Timo Teräsvirta and Tommaso Proietti and the stimulating discussion of Barbara Annicchiarico, Cristina Amado, Monica Billio, Gianna Boero, Alessandra Canepa, Peter Exterkate, Jan G. De Gooijer, Menelaos Karanasos, Alessandra Luati, James Morley, Phil Rothman, and Howell Tong. Dick van Dijk and two anonymous referees are also acknowledged. The author is also grateful to seminar participants at the ECTS 2011 Conference in Villa Modragone, the CFE 2012 Conference in Oviedo, the ICEEE-5th Conference in Genoa, the 8th BMRCQASS Conference on Macro and Financial Economics at Brunel University, and the 2013 Annual Conference of the Royal Statistical Society in Newcastle. This paper was awarded the "James B. Ramsey" Prize for the best paper in Econometrics presented by a Ph.D. student at the 21st Annual Symposium of the Society for Nonlinear Dynamics and Econometrics held at the University of Milan-Bicocca. The author thanks Marcelo C. Medeiros and Barbara Rossi for their MatLab routines, published on their web pages, which were fundamental to the results of this paper. The usual disclaimers apply. This work is in memory of Giancarlo Marini for his great support and encouragement. Finally, the author is in debt to the doctors, assistants, and nurses of the Department of Emathology of Policlinico "S. Matteo" of Pavia, without whose (free) care this paper would remain unfinished. 


\section{References}

Amado, C., Teräsvirta, T., 2013. Modelling volatility by variance decomposition. Journal of Econometrics 175, 142-153.

Amisano, G., Giacomini, R., 2007. Comparing Density Forecasts via Weighted Likelihood Ratio Tests. Journal of Business \& Economic Statistics 25, 177-190.

Anderson, H., Teräsvirta, T., 1992. Characterizing nonlinearities in business cycles using smooth transition autoregressive models. Journal of Applied Econometrics 7 (S1), S119-S136.

Canepa, A., Zanetti Chini, E., 2016. Dynamic asymmetries in house price cycles: A generalized smooth transition model. Journal of Empirical Finance 37, 91-103.

Chan, K., Tong, H., 1986. On estimating thresholds in autoregressive models. Journal of Time Series Analysis 7 (3), 178-190.

Chauvet, M., Piger, J., 2008. A Comparison of the Real-Time Performance of Business Cycle Dating Methods. Journal of Business \& Economic Statistics 26, 42-49.

Clark, T., McCracken, M., 2001. Tests of equal forecast accuracy and encompassing for nested models. Journal of Econometrics 105, 85-110.

Clark, T., Ravazzolo, F., 2015. Macroeconomic Forecasting Performance under Alternative Specification of Time-Varing Volatility. Journal of Applied Econometrics $30,551-575$.

Clements, M., Krolzig, H., 2003. Business Cycle Asymmetries: Characterization and Testing Based on Markov-Switching Autoregressions. Journal of Business Economics \& Statistics 21, 196-211.

Corradi, V., Swanson, N., 2006. Predictive density evaluation. In: Elliott, G., Granger, C., Timmermann, A. (Eds.), Handbook of Economic Forecasting. North Holland. 
Davidson, R., McKinnon, J., 1990. Specification Tests Based on Artificial Regressions. Journal of the American Statistical Association 85, 220-227.

Davies, R., 1977. Hypothesis testing when a nuisance parameter is present only under the alternative. Biometrika 64 (1), 247-254.

De Long, L., Summers, L., 1984. Are Business Cycles Symmetric?

Diebold, F., Mariano, R., 1995. Comparing Predictive Accuracy. Journal of Business \& Economic Statistics 20 (1), 134-144.

Efron, B., Tibshirani, R., 1993. An introduction to the bootstrap. number 57 in monographs on statistics and applied probability.

Engel, J., Haugh, D., Pagan, A., 2005. Some methods for assessing the need for non-linear models in business cycle analysis. International Journal of Forecasting $21,651-662$.

Ferrara, L., Marcellino, M., Mogliani, M., 2015. Macroeconomic forecasting during the Great Recession: The return of non-linearity? International Journal of Forecasting 31, 664-679.

Geweke, J., Amisano, G., 2011. Optimal prediction pools. Journal of Econometrics $164,130-141$.

Giacomini, A., White, H., 2006. Tests for Conditional Predictive Ability. Econometrica 74 (6), 1545-1578.

Giacomini, R., Rossi, B., 2010. Forecast Comparisons in Unstable Environments. Journal of Applied Econometrics 25, 595-620.

Gneiting, T., Raftery, A., 2007. Strictly Proper Scoring Rules, Prediction and Estimation. Journal of the American Statistical Association 102 (477), 359-378. 
González-Rivera, G., 1998. Smooth-Transition GARCH Models. Studies in Nonlinear Dynamics and Econometrics 3 (2), 61-78.

Haggan, V., Ozaki, T., 1981. Modelling nonlinear random vibrations using an amplitude-dependent autoregressive time series model. Biometrika 68 (3), 189196.

Harding, D., Pagan, A., 2006. Synchronization of Cycles. Journal of Econometrics $132,59-79$

Kapetanios, G., Shin, Y., Snell, A., 2003. Testing for a unit root in the nonlinear STAR framework. Journal of Econometrics 112 (2), 359-379.

Kascha, C., Ravazzolo, F., 2010. Combining Inflation Density Forecasts. Journal of Forecasting 29 (1-2), 231-250.

Leybourne, S., Newbold, P., Vougas, D., 1998. Unit roots and smooth transitions. Journal of Time Series Analysis 19 (1), 83-97.

Lundbergh, S., Teräsvirta, T., 2006. A time series model for an exchange rate in a target zone with applications. Journal of Econometrics 131 (1), 579-609.

Lundbergh, S., Teräsvirta, T., van Dick, D., 2003. Time-Varying Smooth Transition Autoregressive Models. Journal of Business and Economic Statistics 21 (1), 10421.

Luukkonen, R., Saikkonen, P., Teräsvirta, T., 1988. Testing linearity against smooth transition autoregressive models. Biometrika 75 (3), 491-499.

McAleer, M., Medeiros, M., 2008. A multiple regime smooth transition Heterogeneous Autoregressive model for long memory and asymmetries. Journal of Econometrics 147, 104-119.

McQueen, G., Thorley, S., 1993. Asymmetric business cycle turning points. Journal of Monetary Economics 31, 341-362. 
Milas, C., Rothman, P., van Dijk, D., Wildasin, D., 2006. Nonlinear time series analysis of business cycles. Emerald Group Publishing Limited.

Neftçi, S., 1984. Are economic time series asymmetric over the business cycle? Journal of Political Economy 92, 307-328.

Rothman, P., 1991. Further evidence on the asymmetric behaviour of unemployment rates over the business cycle. Journal of Macroeconomics 13 (2), 291-298.

Rothman, P., 1998. Forecasting asymmetric unemployment rates. Review of Economics and Statistics 80 (2), 164-168.

Sichel, D., 1993. Business cycle asymmetry: a deeper look. Economic Inquiry 31 (2), $224-236$.

Silvennoinen, A., Teräsvirta, T., 2013. Modelling conditional correlations of asset returns: A smooth transition approach. Econometric ReviewsIn Press.

Sollis, R., Leybourne, S., Newbold, P., 1999. Unit Roots and Asymmetric Smooth Transitions. Journal of Time Series Analysis 20 (6), 671-677.

Sollis, R., Leybourne, S., Newbold, P., 2002. Tests for Symmetric and Asymmetric Nonlinear Mean Reversion in Real Exchange Rates. Journal of Money, Credit and Banking 34 (3), 686-700.

Stock, J., Watson, M., 2012. Disentangling the channels of the 2007Đ2009 recession. Brookings papers on economic activity, Spring 2012, pp. 81-135.

Stukel, T., 1988. Generalized Logistic Models. Journal of American Statistical Association 83 (402), 426-431.

Tashman, L., 2000. Out-of-sample tests of forecasting accuracy: an analysis and review. International Journal of Forecasting 16, 437-450. 
Teräsvirta, T., 1994. Specification, estimation and evaluation of smooth transition autoregressive models. Journal of the American Statistical Association 89 (425), $208-218$.

Teräsvirta, T., Tjøstheim, D., Granger, C., 2010. Modelling Nonlinear Economic Time Series. Oxford University Press, Oxford, New York.

Timmermann, A., 2006. Forecast Combinations. In: Elliot, G., Granger, C., Timmermann, A. (Eds.), Handbook of Economic Forecasting. Elsevier, Amsterdam, pp. 135-196.

Tong, H., 1983. Threshold Models in Non-Linear Time Series Analysis. No. 21 in Lecture Notes in Statistics. Springer-Verlag, New York.

Vougas, D., 2006. On unit root testing with smooth transitions. Computational Statistics and Data Analysis 51, 797-800.

West, K., 1996. Asymptotic inference about predictive ability. Econometrica 64, 1067-1084. 


\section{Tables and Graphs}

Figure 1: The US quarterly data

(a) Data in levels
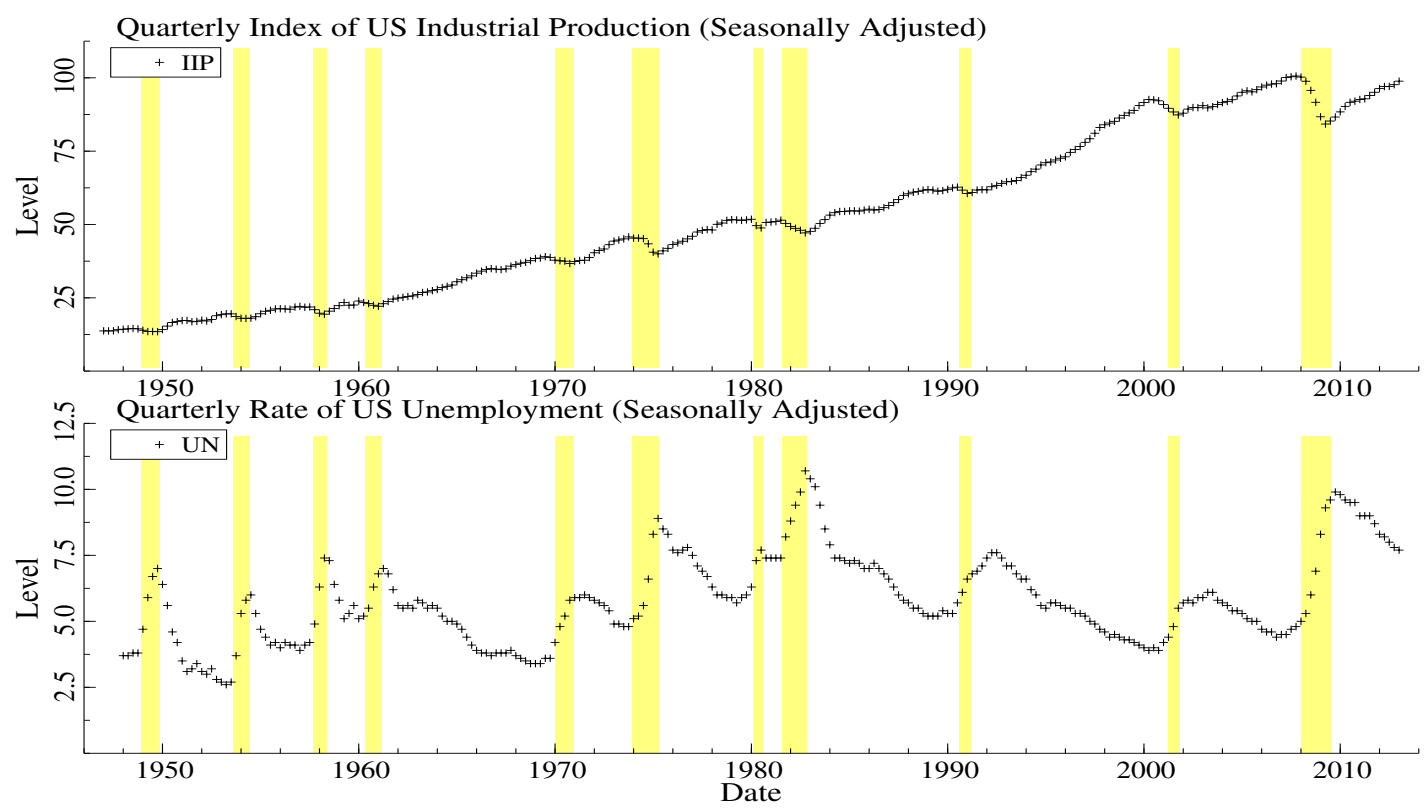

(b) Data in growth rates
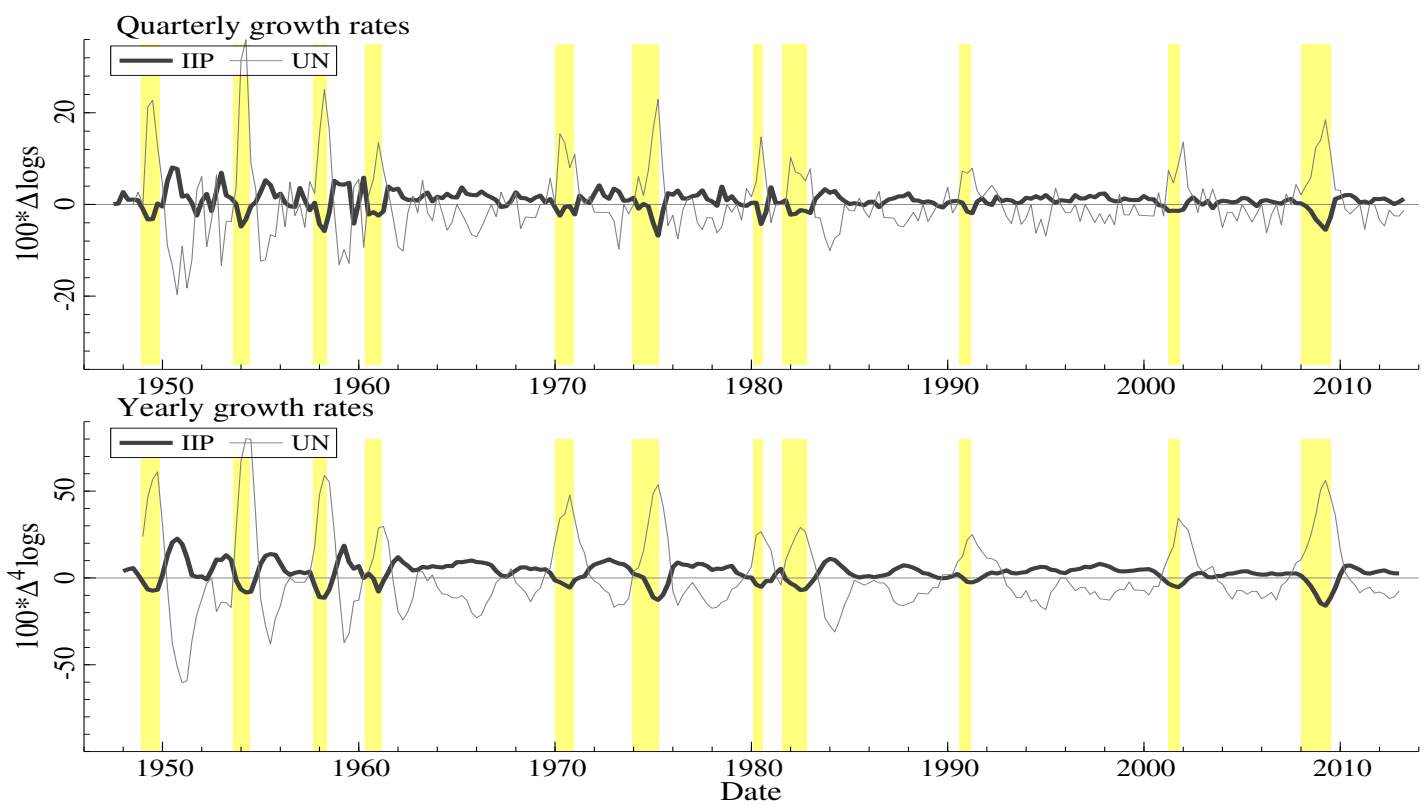

NOTE: This figure plots the quarterly data on US index of industrial production (IIP) and unemployment (UN) used to illustrate the performances of GSTAR model in Section 4. In particular, panel (a) plots the series in levels, while panel (b) plots the same series in quarterly (upper subpanel) and yearly growth rates (lower sub-panel), respectively. The bands in yellow are the NBER recession dates. 
Figure 2: Types of asymmetry in the transition functions

(a) Asymmetric transition functions
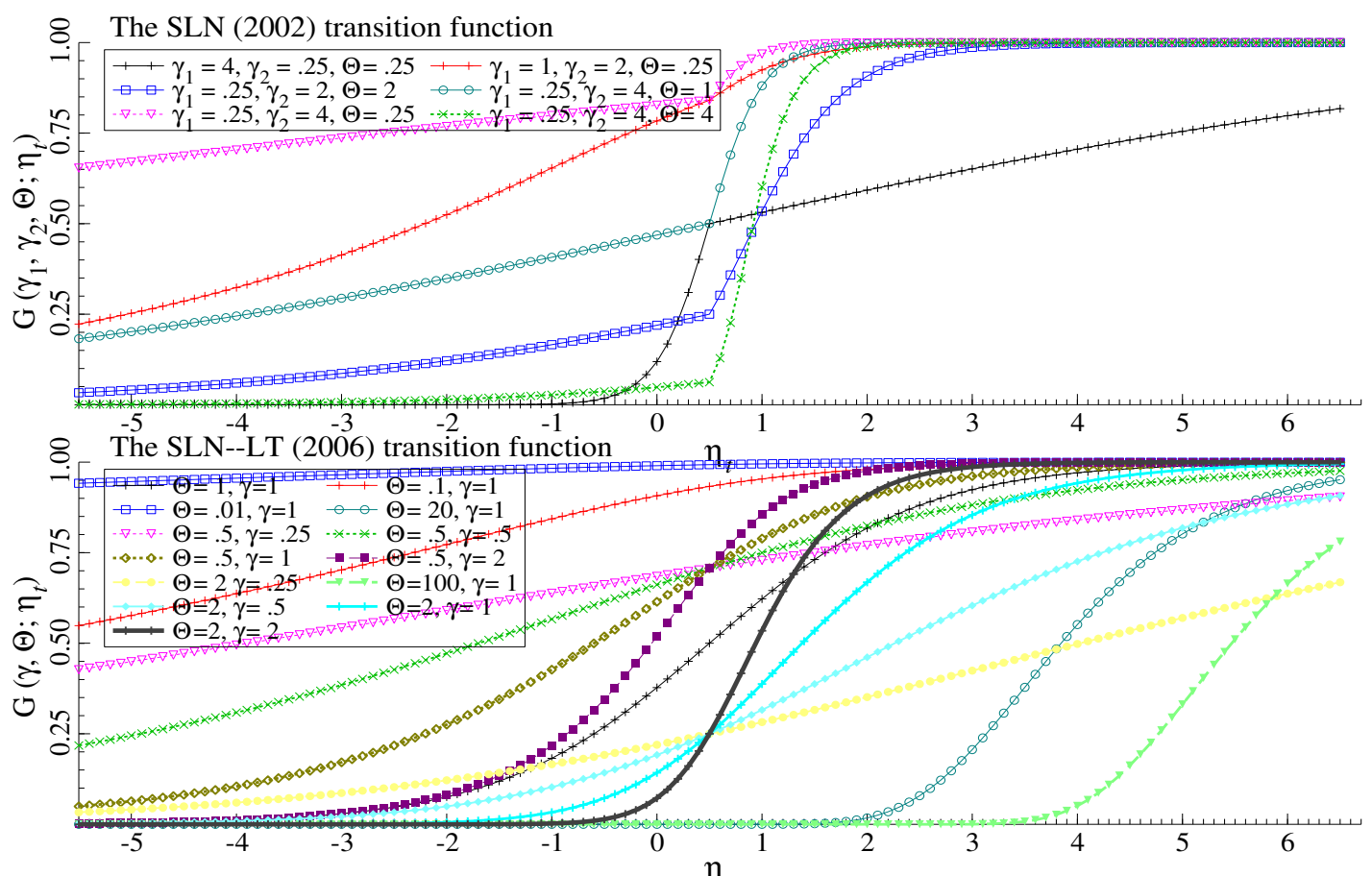

(b) Dynamically asymmetric transition functions

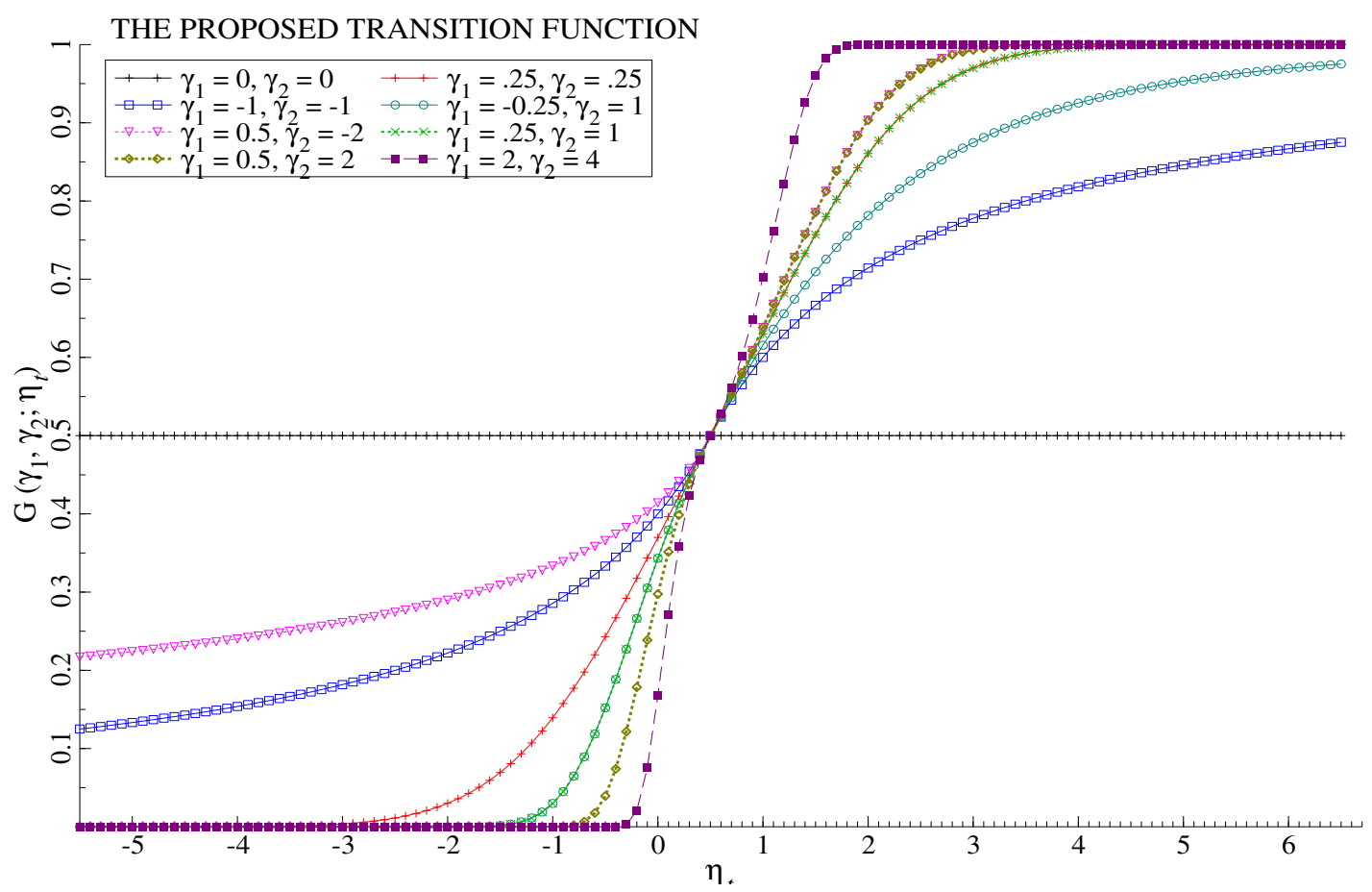

NOTE: This figure illustrates several different parametrizations of asymmetric transition function for a STAR model. In particular, panel (a) plots the asymmetric ones currently available in literature, namely the Sollis et al. (2002) and the Sollis et al. (1999)-Lundbergh and Teräsvirta (2006) models; panel (b) plots the dynamically asymmetric transition function here proposed. 
Figure 3: Taxonomy of the dynamic asymmetry in business cycle
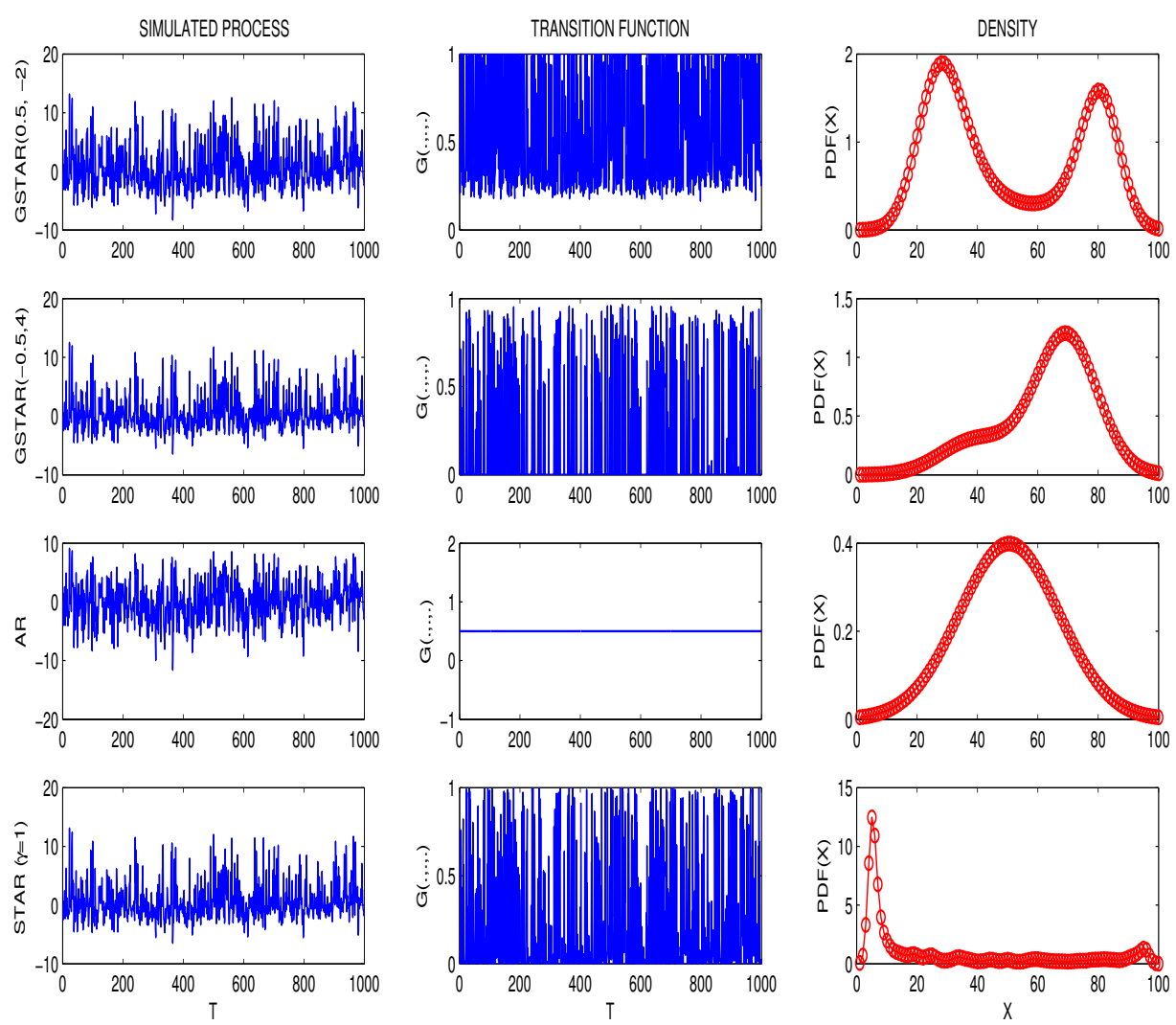

NOTE: The figure depicts four stylized business cycles. The first row presents the representation of a cycle simulated by a GSTAR with $\gamma_{1}>\gamma_{2}$ corresponding to strong deep and low steep; the second row a cycle simulated by a GSTAR with $\gamma_{2}>\gamma_{1}$, where deepness is moderate and steep is high; the third row a cycle corresponding to a liner AR; the fourth row a purely steep cycle simulated by a STAR model. For each row, the first column (denoted "Simulated Process") displays the time path of the time series $y_{t}$, the second column (denoted "Transition Function") shows the time path of the corresponding $G(\cdot, \cdot)$; finally, the third column (denoted "Density") gives the estimated density of the nonlinear part of the process, here denoted $X$, where $X=\hat{\boldsymbol{\theta}}^{\prime} \mathbf{z}_{\mathbf{t}} \times G(\cdot, \cdot)$. 
Figure 4: Estimated transition functions specifications for quarterly IIP

(a) Yearly growth rate
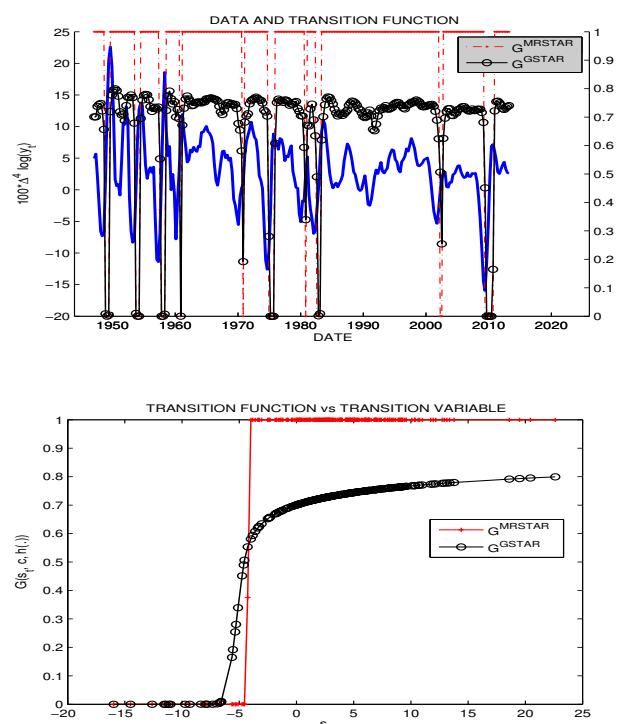

(b) Quarterly growth rate
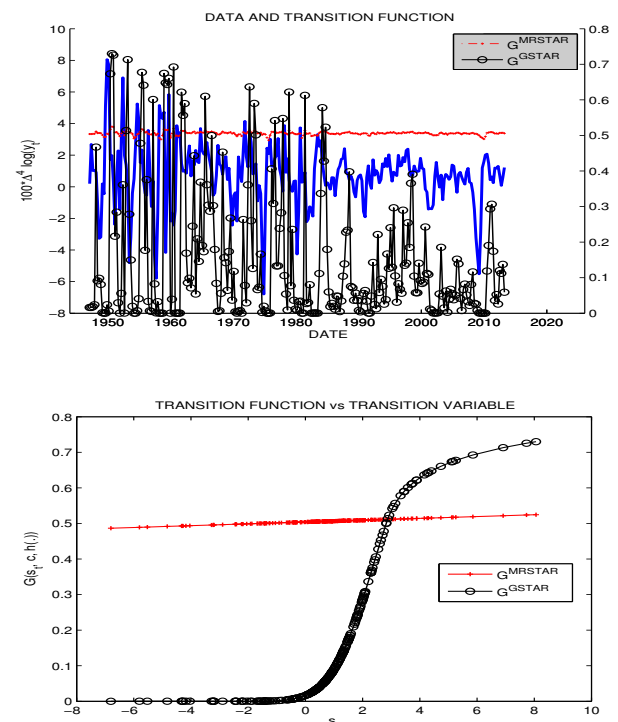

NOTE: This figures plots the estimated transition functions of MRSTAR and GSTAR models estimated from quarterly IIP series. The left-had side shows the results for yearly growth rates, while right-had side the monthly growth rates; upper panels plots data with transition functions versus time, while in bottom panels the same transitions are shown versus the transition variable.

Figure 5: Estimated transition functions specifications for monthly IIP

(a) Yearly growth rate
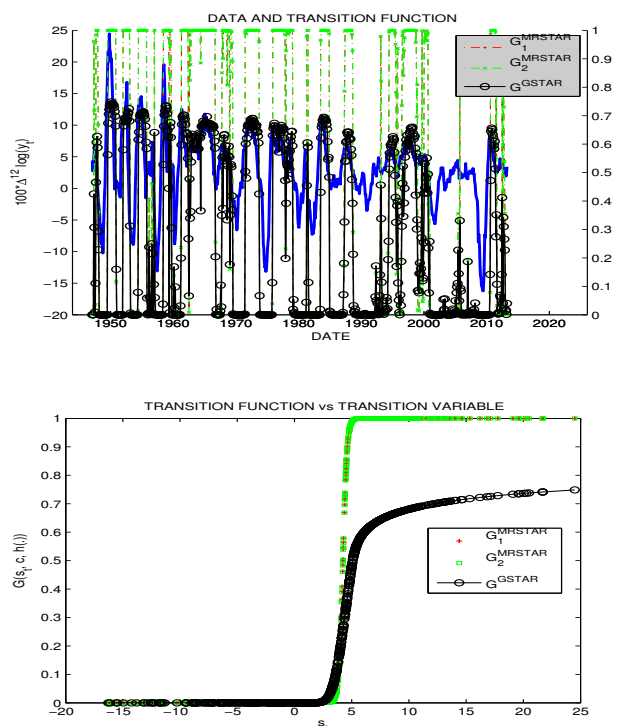

(b) Monthly growth rate
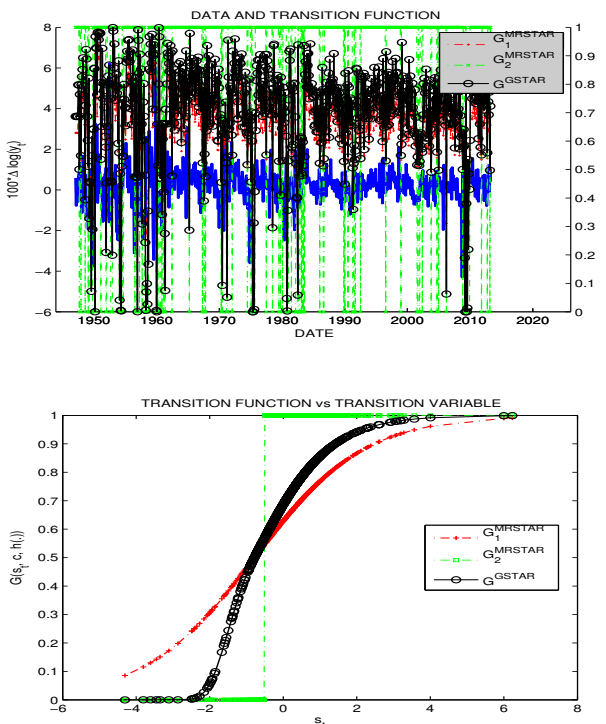

NOTE: This figures plots the estimated transition functions of MRSTAR and GSTAR models estimated from monthly IIP series. The left-had side shows the results for yearly growth rates, while right-had side the monthly growth rates; upper panels plots data with transition functions versus time, while in bottom panels the same transitions are shown versus the transition variable. 
Figure 6: Estimated transition functions for quarterly UN

(a) Yearly growth rate
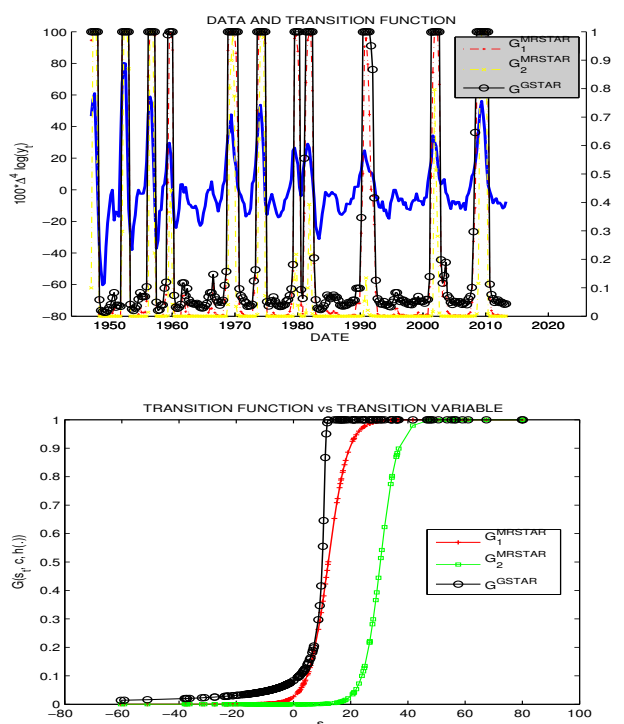

(b) Quarterly growth rate
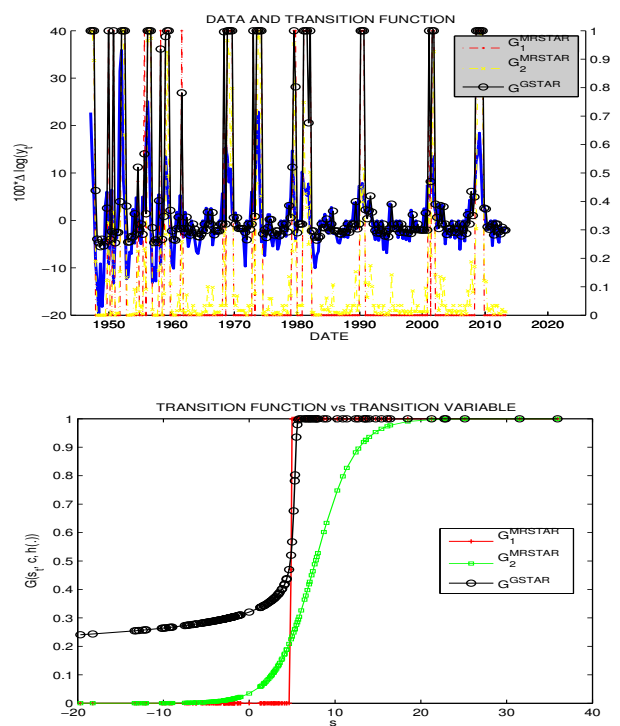

NOTE: This figures plots the estimated transition functions of MRSTAR and GSTAR models estimated from quarterly UN series. The left-had side shows the results for yearly growth rates, while right-had side the monthly growth rates; upper panels plots data with transition functions versus time, while in bottom panels the same transitions are shown versus the transition variable.

Figure 7: Estimated transition functions for monthly UN

(a) Yearly growth rate
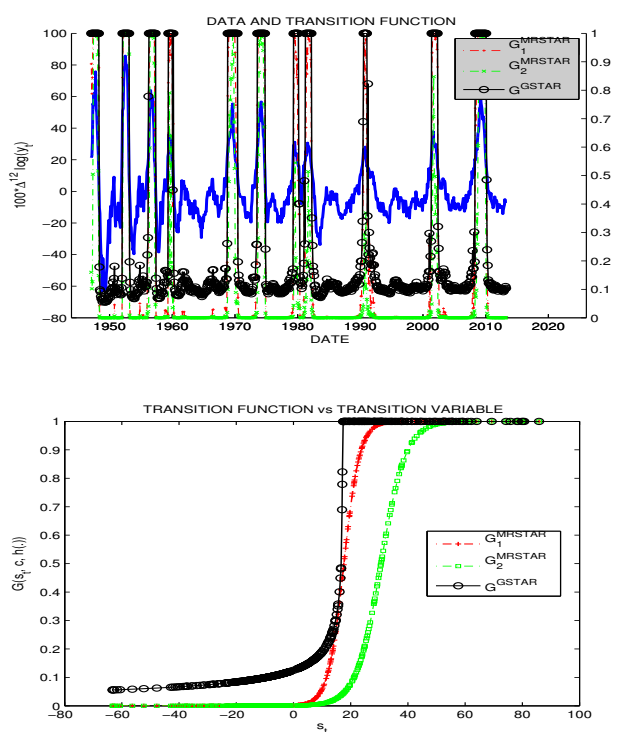

(b) Monthly growth rate
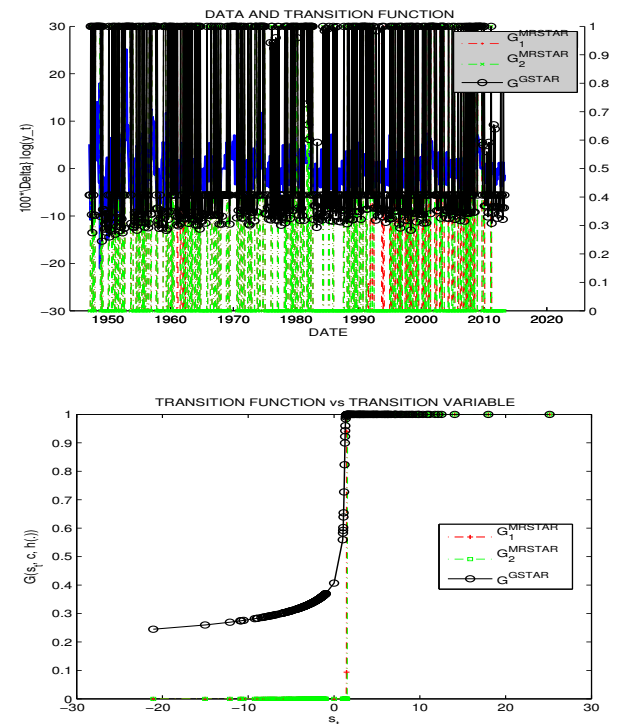

NOTE: This figures plots the estimated transition functions of MRSTAR and GSTAR models estimated from monthly UN series. The left-had side shows the results for yearly growth rates, while right-had side the monthly growth rates; upper panels plots data with transition functions versus time, while in bottom panels the same transitions are shown versus the transition variable. 


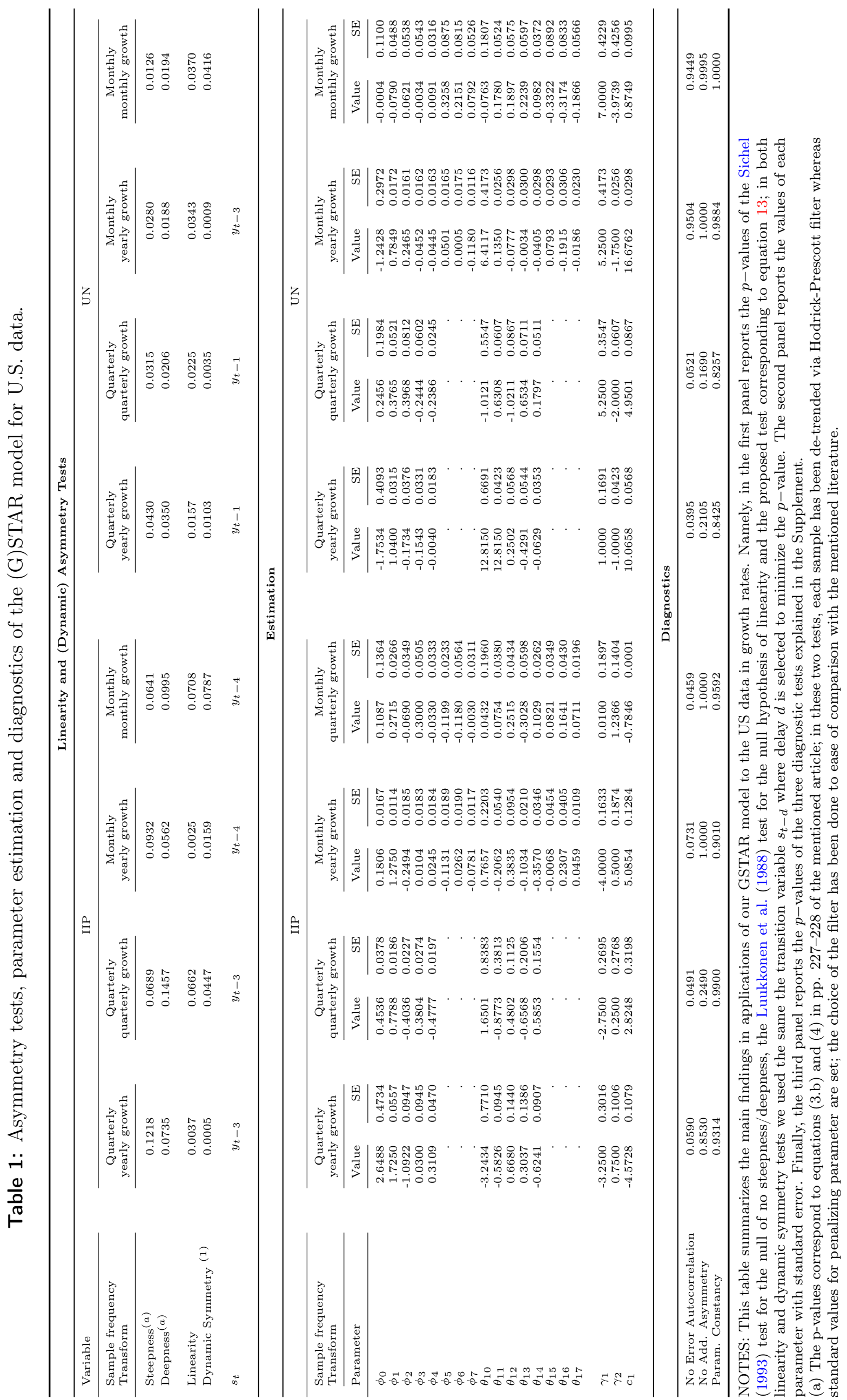


Table 2: GSTAR versus linear and nonlinear symmetric models: comparison of predictive performances in quarterly U.S. data.

\begin{tabular}{|c|c|c|c|c|c|c|c|}
\hline \multirow[b]{2}{*}{ Forecast horizon } & \multicolumn{7}{|c|}{ Point predictive performances } \\
\hline & \multirow[t]{2}{*}{$\begin{array}{l}\text { Forecast Error } \\
\text { Measure } \\
\end{array}$} & \multicolumn{2}{|c|}{$\mathrm{AR}$} & \multicolumn{2}{|c|}{$(\mathrm{MR}) \mathrm{STAR}$} & \multicolumn{2}{|c|}{ GSTAR } \\
\hline & & IIP & UN & IIP & UN & IIP & UN \\
\hline 1 & & -0.1823 & -0.8710 & -0.1417 & -0.0456 & -0.1070 & -0.8698 \\
\hline 2 & MFE & -0.1928 & -0.9323 & -0.1437 & -0.0525 & -0.1094 & -0.9255 \\
\hline 4 & & -01969 & -0.9528 & -0.1377 & -0.0831 & -0.1076 & -1.0273 \\
\hline 8 & & -0.1991 & -1.1300 & -0.1616 & -0.1271 & -0.1349 & -1.1687 \\
\hline 1 & & 0.0077 & 0.0669 & 0.0030 & 0.0676 & 0.0032 & 0.0630 \\
\hline 2 & sMAE & 0.0079 & 0.0692 & 0.0030 & 0.0745 & 0.0032 & 0.0665 \\
\hline 4 & & 0.0085 & 0.0708 & 0.0031 & 0.0792 & 0.0033 & 0.0626 \\
\hline 8 & & 0.0087 & 0.0732 & 0.0032 & 0.0737 & 0.0034 & 0.0650 \\
\hline 1 & & 1.0000 & 1.0000 & 1.3433 & 0.4214 & 1.2074 & 0.3063 \\
\hline 2 & mRAE & 1.0000 & 1.0000 & 1.4061 & 0.3997 & 1.3400 & 0.3099 \\
\hline 4 & & 1.0000 & 1.0000 & 1.6696 & 0.4139 & 1.6212 & 0.3559 \\
\hline 8 & & 1.0000 & 1.0000 & 2.6900 & 0.4817 & 2.5691 & 0.3879 \\
\hline 1 & & 0.1154 & 1.7223 & 0.1151 & 1.5889 & 0.1136 & 1.0372 \\
\hline 2 & RMSFE & 0.1159 & 1.8310 & 0.1158 & 1.5991 & 0.1144 & 1.0427 \\
\hline 4 & & 0.1172 & 1.8969 & 0.1171 & 1.6197 & 0.1158 & 1.0542 \\
\hline 8 & & 0.1224 & 1.9344 & 0.1197 & 1.6626 & 0.1285 & 1.0805 \\
\hline \multicolumn{8}{|c|}{ Density predictive performances } \\
\hline 1 & & 0.0104 & 0.0179 & 0.0105 & 0.0176 & 0.0110 & 0.0166 \\
\hline 2 & $\operatorname{LogS}$ & 0.0105 & 0.0182 & 0.0105 & 0.0176 & 0.0109 & 0.0166 \\
\hline 4 & & 0.0107 & 0.0182 & 0.0106 & 0.0175 & 0.0112 & 0.0172 \\
\hline 8 & & 0.0107 & 0.0184 & 0.0110 & 0.0175 & 0.0123 & 0.0172 \\
\hline 1 & & 0.2138 & 0.0651 & 0.2202 & 0.0647 & 0.2131 & 0.0637 \\
\hline 2 & QS & 0.2201 & 0.0654 & 0.2191 & 0.0650 & 0.2111 & 0.0639 \\
\hline 4 & & 0.2252 & 0.0659 & 0.2210 & 0.0650 & 0.2113 & 0.0639 \\
\hline 8 & & 0.2252 & 0.0662 & 0.2219 & 0.0653 & 0.2152 & 0.0642 \\
\hline 1 & & 7.0738 & 31.5422 & 7.0256 & 31.5500 & 7.0655 & 31.5100 \\
\hline 2 & CRPS & 7.1141 & 31.7299 & 7.0750 & 31.7077 & 7.1123 & 31.7377 \\
\hline 4 & & 7.2140 & 32.2400 & 7.1740 & 32.0356 & 7.2132 & 32.0383 \\
\hline 8 & & 7.4200 & 32.7444 & 7.3708 & 32.7216 & 7.4222 & 32.7219 \\
\hline 1 & & 0.2092 & 0.0421 & 0.2088 & 0.0382 & 0.2038 & 0.0376 \\
\hline 2 & qS & 0.2103 & 0.0489 & 0.2085 & 0.0449 & 0.2036 & 0.0443 \\
\hline 4 & & 0.2118 & 0.0528 & 0.2066 & 0.0524 & 0.2020 & 0.0584 \\
\hline 8 & & 0.2118 & 0.0772 & 0.2048 & 0.0803 & 0.2004 & 0.0803 \\
\hline
\end{tabular}

NOTES: This table reports the point (first panel) and density (second panel) predictive performances of linear (AR) nonlinear symmetric (MRSTAR) and dynamic asymmetric (GSTAR) models according to different measures and forecasting horizons with testing period from 1982:Q3 to 2013Q1 for IIP and from 1983:Q3 to 2013Q1 for UN. The smallest values for each forecasting horizon are in bold. 
Table 3: GSTAR versus linear and nonlinear symmetric models: comparison of predictive performances in monthly U.S. data

\begin{tabular}{|c|c|c|c|c|c|c|c|}
\hline \multirow{3}{*}{ Forecast horizon } & \multicolumn{7}{|c|}{ Point predictive performances } \\
\hline & \multirow[t]{2}{*}{$\begin{array}{l}\text { Forecast Error } \\
\text { Measure }\end{array}$} & \multicolumn{2}{|c|}{$\mathrm{AR}$} & \multicolumn{2}{|c|}{ (MR)STAR } & \multicolumn{2}{|c|}{ GSTAR } \\
\hline & & IIP & UN & IIP & UN & IIP & UN \\
\hline 1 & & -0.0011 & 0.0009 & -0.0065 & 0.0156 & -0.0093 & 0.0095 \\
\hline 3 & MFE & -0.1108 & 0.0340 & -0.0071 & 0.0168 & -0.0098 & 0.0108 \\
\hline 6 & & -0.3024 & 0.0607 & -0.0072 & 0.0150 & -0.0099 & 0.0093 \\
\hline 12 & & -1.4908 & 0.1117 & -0.0068 & 0.0163 & -0.0094 & 0.0107 \\
\hline 1 & & 0.0011 & 0.0058 & 0.0011 & 0.0061 & 0.0011 & 0.0060 \\
\hline 3 & sMAE & 0.0037 & 0.0087 & 0.0011 & 0.0059 & 0.0011 & 0.0058 \\
\hline 6 & & 0.0070 & 0.0128 & 0.0012 & 0.0056 & 0.0012 & 0.0055 \\
\hline 12 & & 0.0124 & 0.0190 & 0.0012 & 0.0050 & 0.0012 & 0.0049 \\
\hline 1 & & 1.0000 & 1.0000 & 1.0324 & 1.0224 & 1.0290 & 1.0107 \\
\hline 3 & mRAE & 1.0000 & 1.0000 & 1.1560 & 1.0552 & 1.1241 & 1.0369 \\
\hline 6 & & 1.0000 & 1.0000 & 1.4886 & 1.2189 & 1.4546 & 1.1693 \\
\hline 12 & & 1.0000 & 1.0000 & 2.0115 & 1.4965 & 2.1142 & 1.5085 \\
\hline 1 & & 0.0698 & 0.2856 & 0.0051 & 0.0178 & 0.0042 & 0.0176 \\
\hline 3 & RMSFE & 0.2894 & 0.4234 & 0.0052 & 0.0179 & 0.0043 & 0.0177 \\
\hline 6 & & 0.7552 & 0.5915 & 0.0052 & 0.0181 & 0.0046 & 0.0179 \\
\hline \multirow[t]{2}{*}{12} & & 0.7638 & 0.8545 & 0.0055 & 0.0184 & 0.0049 & 0.0182 \\
\hline & \multicolumn{7}{|c|}{ Density predictive performances } \\
\hline 1 & & 0.0020 & 0.0031 & 0.0017 & 0.0033 & 0.0014 & 0.0036 \\
\hline 3 & $\operatorname{LogS}$ & 0.0018 & 0.0034 & 0.0017 & 0.0036 & 0.0014 & 0.0035 \\
\hline 6 & & 0.0021 & 0.0035 & 0.0017 & 0.0036 & 0.0016 & 0.0035 \\
\hline 12 & & 0.0032 & 0.0036 & 0.0017 & 0.0036 & 0.0021 & 0.0035 \\
\hline 1 & & 2.1568 & 0.7986 & 2.1303 & 0.8511 & 2.1689 & 0.8683 \\
\hline 3 & QS & 2.1644 & 0.7998 & 2.1386 & 0.8537 & 2.1821 & 0.8698 \\
\hline 6 & & 2.1665 & 0.8125 & 2.1391 & 0.8550 & 2.1895 & 0.8698 \\
\hline 12 & & 2.2024 & 0.6741 & 2.1479 & 0.6412 & 2.1921 & 0.6354 \\
\hline 1 & & 0.2142 & 1.6750 & 0.2170 & 1.6090 & 0.2201 & 1.6094 \\
\hline 3 & CRPS & 0.2161 & 1.6850 & 0.2189 & 1.6193 & 0.2224 & 1.6197 \\
\hline 6 & & 0.2188 & 1.6983 & 0.2212 & 1.6317 & 0.2252 & 1.6344 \\
\hline 12 & & 0.2255 & 1.7295 & 0.2279 & 1.6594 & 0.2318 & 1.6617 \\
\hline 1 & & 0.0094 & -0.0713 & 0.0089 & -0.0727 & 0.0087 & -0.0723 \\
\hline 3 & $\mathrm{qS}$ & 0.0093 & -0.0706 & 0.0085 & -0.0721 & 0.0086 & -0.0717 \\
\hline 6 & & 0.0092 & -0.0695 & 0.0083 & -0.0708 & 0.0085 & -0.0705 \\
\hline 12 & & 0.0087 & -0.0667 & 0.0071 & -0.0682 & 0.0080 & -0.0679 \\
\hline
\end{tabular}

NOTES: This table reports the point (first panel) and density (second panel) predictive performances of linear (AR) nonlinear symmetric (MRSTAR) and dynamic asymmetric (GSTAR) models according to different measures and forecasting horizons with testing period from 1982:M9 to 2013:M3 for IIP and from 1983:M9 to 2013:M3 for UN. The smallest values for each forecasting horizon are in bold. 
Table 4: GSTAR versus linear and nonlinear symmetric models under parameter uncertainty: comparison of predictive performances in quarterly U.S. data

\begin{tabular}{|c|c|c|c|c|c|c|c|}
\hline \multirow{3}{*}{ Forecast horizon } & \multicolumn{7}{|c|}{ Point predictive performances } \\
\hline & \multirow[t]{2}{*}{$\begin{array}{l}\text { Forecast Error } \\
\text { Measure }\end{array}$} & \multicolumn{2}{|c|}{ AR } & \multicolumn{2}{|c|}{ (MR)STAR } & \multicolumn{2}{|c|}{ GSTAR } \\
\hline & & IIP & UN & IIP & UN & IIP & UN \\
\hline 1 & \multirow{4}{*}{ MFE } & -2.4600 & -1.2362 & -2.4670 & -1.1701 & -2.4685 & -1.3015 \\
\hline 2 & & -2.4659 & -1.2930 & -2.4632 & -1.2361 & -2.4686 & -1.3664 \\
\hline 4 & & -2.4682 & -1.4295 & -2.4608 & -1.3745 & -2.4427 & -1.5040 \\
\hline 8 & & -2.4682 & -1.6072 & -2.4586 & -1.5868 & -2.4391 & -1.7101 \\
\hline 1 & \multirow{4}{*}{ sMAE } & 0.0204 & 0.1101 & 0.0201 & 0.1266 & 0.0200 & 0.1120 \\
\hline 2 & & 0.0204 & 0.1062 & 0.0202 & 0.1208 & 0.0202 & 0.1074 \\
\hline 4 & & 0.0210 & 0.0975 & 0.0205 & 0.1103 & 0.0205 & 0.0992 \\
\hline 8 & & 0.0217 & 0.0902 & 0.0215 & 0.0992 & 0.0214 & 0.0907 \\
\hline 1 & \multirow{4}{*}{ mRAE } & 1.0000 & 1.0000 & 1.0000 & 1.0035 & 1.0001 & 1.0017 \\
\hline 2 & & 1.0000 & 1.0000 & 1.0042 & 1.0011 & 1.0038 & 1.0025 \\
\hline 4 & & 1.0000 & 1.0000 & 1.0069 & 1.0016 & 1.0071 & 1.0025 \\
\hline 8 & & 1.0000 & 1.0000 & 1.0037 & 1.0021 & 1.0039 & 1.0034 \\
\hline 1 & \multirow{4}{*}{ RMSFE } & 0.3707 & 1.1720 & 0.3756 & 1.2322 & 0.3751 & 1.2337 \\
\hline 2 & & 0.3723 & 1.1786 & 0.3775 & 1.2387 & 0.3771 & 1.2403 \\
\hline 4 & & 0.3761 & 1.1908 & 0.3805 & 1.2518 & 0.3800 & 1.2535 \\
\hline \multirow[t]{2}{*}{8} & & 0.3835 & 1.2201 & 0.3888 & 1.2820 & 0.3882 & 1.2841 \\
\hline & \multicolumn{7}{|c|}{ Density predictive performances } \\
\hline 1 & \multirow{4}{*}{$\operatorname{LogS}$} & 0.0136 & 0.0169 & 0.0135 & 0.0176 & 0.0130 & 0.0181 \\
\hline 2 & & 0.0135 & 0.0169 & 0.0134 & 0.0176 & 0.0130 & 0.0181 \\
\hline 4 & & 0.0135 & 0.0168 & 0.0133 & 0.0175 & 0.0129 & 0.0180 \\
\hline 8 & & 0.0133 & 0.0167 & 0.0131 & 0.0175 & 0.0128 & 0.0180 \\
\hline 1 & \multirow{4}{*}{ QS } & 0.2244 & 0.0635 & 0.2242 & 0.0634 & 0.2240 & 0.0639 \\
\hline 2 & & 0.2243 & 0.0638 & 0.2240 & 0.0637 & 0.2238 & 0.0639 \\
\hline 4 & & 0.2243 & 0.0640 & 0.2244 & 0.0637 & 0.2239 & 0.0647 \\
\hline 8 & & 0.2245 & 0.0652 & 0.2245 & 0.0652 & 0.2244 & 0.0647 \\
\hline 1 & \multirow{4}{*}{ CRPS } & 6.2854 & 30.1794 & 6.0036 & 31.5500 & 5.9310 & 30.4696 \\
\hline 2 & & 6.3291 & 30.3430 & 6.0443 & 31.7077 & 5.9689 & 30.6087 \\
\hline 4 & & 6.4230 & 30.6666 & 6.1338 & 32.0356 & 6.0561 & 30.9277 \\
\hline 8 & & 6.6149 & 31.3386 & 6.3109 & 32.7216 & 6.2307 & 31.6032 \\
\hline 1 & \multirow{4}{*}{ qS } & 0.0440 & 0.0093 & 0.0463 & 0.0075 & 0.2330 & 0.0509 \\
\hline 2 & & 0.0440 & 0.0105 & 0.0463 & 0.0089 & 0.2327 & 0.0576 \\
\hline 4 & & 0.0437 & 0.0133 & 0.0460 & 0.0117 & 0.2311 & 0.0716 \\
\hline 8 & & 0.0432 & 0.0170 & 0.0456 & 0.0161 & 0.2291 & 0.0928 \\
\hline
\end{tabular}

NOTES: This table reports the point (first panel) and density (second panel) predictive performances of linear (AR) nonlinear symmetric (MRSTAR) and dynamic asymmetric (GSTAR) models accounting for uncertainty via block-boostrap algorithm according to different measures and forecasting horizons with testing period from 1982:Q3 to 2013Q1 for IIP and from 1983:Q3 to $2013 \mathrm{Q} 1$ for UN. The smallest values for each forecasting horizon are in bold. 
Table 5: GSTAR versus linear and nonlinear symmetric models under parameter uncertainty: comparison of predictive performances in monthly U.S. data

\begin{tabular}{|c|c|c|c|c|c|c|c|}
\hline \multirow{3}{*}{ Forecast horizon } & \multicolumn{7}{|c|}{ Point predictive performances } \\
\hline & \multirow[t]{2}{*}{$\begin{array}{l}\text { Forecast Error } \\
\text { Measure }\end{array}$} & \multicolumn{2}{|c|}{$\mathrm{AR}$} & \multicolumn{2}{|c|}{$(\mathrm{MR}) \mathrm{STAR}$} & \multicolumn{2}{|c|}{ GSTAR } \\
\hline & & IIP & UN & $\mathrm{I} 5 \mathrm{P}$ & UN & IIP & $\mathrm{UN}$ \\
\hline 1 & & 0.7134 & -0.0309 & 0.5894 & -0.0308 & 0.5781 & 0.0008 \\
\hline 3 & MFE & 0.8400 & -0.0006 & 0.7628 & -0.0205 & 0.5657 & 0.0465 \\
\hline 6 & & 0.5048 & -0.0020 & 0.7489 & -0.0166 & 0.6159 & -0.0074 \\
\hline 12 & & 0.8711 & -0.0530 & 0.8340 & -0.0129 & 0.7099 & -0.0090 \\
\hline 1 & & 0.0057 & 0.0308 & 0.0020 & 0.0144 & 0.0058 & 0.0272 \\
\hline 3 & sMAE & 0.0056 & 0.0271 & 0.0020 & 0.0137 & 0.0056 & 0.0318 \\
\hline 6 & & 0.0056 & 0.0253 & 0.0021 & 0.0131 & 0.0061 & 0.0258 \\
\hline 12 & & 0.0059 & 0.0261 & 0.0022 & 0.0124 & 0.0056 & 0.0231 \\
\hline 1 & & 1.0000 & 1.0000 & 0.6814 & 0.0063 & 0.6143 & 0.0173 \\
\hline 3 & mRAE & 1.0000 & 1.0000 & 1.1191 & 0.1223 & 0.9089 & 0.1175 \\
\hline 6 & & 1.0000 & 1.0000 & 3.0881 & 0.1245 & 0.9029 & 0.1701 \\
\hline 12 & & 1.0000 & 1.0000 & 5.1183 & 0.1421 & 0.9937 & 0.2119 \\
\hline 1 & & 0.0641 & 0.0780 & 0.0368 & 0.0603 & 0.0598 & 0.0774 \\
\hline 3 & RMSFE & 0.0662 & 0.0789 & 0.0329 & 0.0604 & 0.0543 & 0.0781 \\
\hline 6 & & 0.0489 & 0.0784 & 0.0425 & 0.0606 & 0.0510 & 0.0786 \\
\hline \multirow[t]{2}{*}{12} & & 0.0706 & 0.0797 & 0.0483 & 0.0612 & 0.0625 & 0.0800 \\
\hline & \multicolumn{7}{|c|}{ Density predictive performances } \\
\hline 1 & & 0.0019 & 0.0036 & -0.0001 & 0.0034 & 0.0020 & 0.0037 \\
\hline 3 & $\operatorname{LogS}$ & 0.0018 & 0.0035 & -0.0001 & 0.0034 & 0.0023 & 0.0039 \\
\hline 6 & & 0.0018 & 0.0035 & -0.0001 & -0.0166 & 0.0029 & 0.0040 \\
\hline 12 & & 0.0018 & 0.0037 & -0.0001 & 0.0034 & 0.0038 & 0.0044 \\
\hline 1 & & 2.2142 & 0.8870 & 2.5261 & 1.0937 & 2.2040 & 0.8763 \\
\hline 3 & QS & 2.2146 & 0.8879 & 2.4917 & 1.0995 & 2.2086 & 0.8769 \\
\hline 6 & & 2.2234 & 0.8876 & 2.1880 & 1.0929 & 2.2100 & 0.8773 \\
\hline 12 & & 2.2250 & 0.8920 & 2.2437 & 1.0095 & 2.2195 & 0.8819 \\
\hline 1 & & -0.4926 & 1.6349 & 0.1968 & 0.8900 & 0.2219 & 1.6349 \\
\hline 3 & CRPS & 0.2241 & 1.6431 & 0.1974 & 0.8922 & 0.2241 & 1.6431 \\
\hline 6 & & 0.2273 & 1.6579 & 0.1982 & 0.8954 & 0.2273 & 1.6579 \\
\hline 12 & & 0.2337 & 1.6865 & 0.1999 & 0.9018 & 0.2337 & 1.6865 \\
\hline 1 & & 0.0017 & -0.0157 & -0.0030 & -0.0142 & 0.0019 & -0.0145 \\
\hline 3 & $\mathrm{qS}$ & 0.0017 & -0.0155 & -0.0030 & -0.0141 & 0.0019 & -0.0144 \\
\hline 6 & & 0.0019 & -0.0153 & -0.0030 & -0.0140 & 0.0020 & -0.0141 \\
\hline 12 & & 0.0021 & -0.0146 & -0.0031 & -0.0138 & 0.0025 & -0.0136 \\
\hline
\end{tabular}

NOTES: This table reports the point (first panel) and density (second panel) predictive performances of linear (AR) nonlinear symmetric (MRSTAR) and dynamic asymmetric (GSTAR) models accounting for uncertainty via block-boostrap algorithm according to different measures and forecasting horizons with testing period from 1982:M9 to 2013:M3 for IIP and from 1983:M9 to 2013:M3 for UN. The smallest values for each forecasting horizon are in bold. 
Table 6: Comparison of predictive ability tests of GSTAR versus linear and symmetric models in quarterly U.S. data

\begin{tabular}{|c|c|c|c|c|c|c|c|c|}
\hline \multirow{3}{*}{$\begin{array}{r}\text { SERIES } \\
\mathrm{h}\end{array}$} & \multicolumn{4}{|c|}{ MR-STAR vs AR } & \multicolumn{4}{|c|}{ GSTAR vs MR-STAR } \\
\hline & \multicolumn{2}{|c|}{ IIP } & \multicolumn{2}{|c|}{$\mathrm{UN}$} & \multicolumn{2}{|c|}{ IIP } & \multicolumn{2}{|c|}{ UN } \\
\hline & \multicolumn{8}{|c|}{ Diebold-Mariano } \\
\hline 1 & \multicolumn{2}{|c|}{0.1196} & \multicolumn{2}{|c|}{0.1668} & \multicolumn{2}{|c|}{0.1165} & \multicolumn{2}{|c|}{0.2260} \\
\hline 2 & \multicolumn{2}{|c|}{0.0890} & \multicolumn{2}{|c|}{0.2550} & \multicolumn{2}{|c|}{0.1170} & \multicolumn{2}{|c|}{0.2259} \\
\hline 4 & \multicolumn{2}{|c|}{0.1011} & \multicolumn{2}{|c|}{0.2540} & \multicolumn{2}{|c|}{0.1978} & \multicolumn{2}{|c|}{0.2841} \\
\hline 8 & \multicolumn{2}{|c|}{0.2104} & \multicolumn{2}{|c|}{0.3054} & \multicolumn{2}{|c|}{0.2080} & \multicolumn{2}{|c|}{0.3270} \\
\hline $\mathrm{h}$ & \multicolumn{8}{|c|}{ Giacomini-Whight } \\
\hline 1 & \multicolumn{2}{|c|}{0.0610} & \multicolumn{2}{|c|}{0.0568} & \multicolumn{2}{|c|}{0.0327} & 0.01 & \\
\hline 2 & 0.08 & & 0.07 & & 0.13 & & 0.0 & \\
\hline 4 & 0.12 & & 0.00 & & 0.20 & & $0.2 s$ & \\
\hline 8 & 0.33 & & 0.28 & & 0.3 & & 0.5 & \\
\hline Scoring Rule & & & & Amis & comini & & & \\
\hline $\mathrm{h}$ & If & & UI & & II & & $\mathrm{U}$ & \\
\hline QSR & $t$-statistic & $p$-val & $t$-statistic & $p$-val & $t$-statistic & $p$-val & $t$-statistic & $p$-val \\
\hline 1 & $1.9 e^{e-5}$ & 0.4600 & 0.0015 & 0.4994 & $2.9 e^{4}$ & 0.5567 & $6.0 e^{-4}$ & 0.4999 \\
\hline 2 & $1.9 e^{-5}$ & 0.4975 & 0.0015 & 0.4994 & $2.9 e^{4}$ & 0.5553 & $6.0 e^{-4}$ & 0.4999 \\
\hline 4 & $2.9 e-7$ & 0.5120 & 0.0016 & 0.4993 & $2.9 e^{4}$ & 0.5312 & $4.1 e^{-5}$ & 0.5998 \\
\hline 8 & $2.9 e^{-9}$ & 0.5169 & 0.0016 & 0.4993 & $2.3 e^{4}$ & 0.5622 & $9.2 e^{-5}$ & 1.0000 \\
\hline $\log S$ & & & & & & & & \\
\hline 1 & -16.2383 & 1.0000 & -2017.1657 & 1.0000 & -0.3535 & 0.6380 & $-3.9 \mathrm{e}^{3}$ & 1.0000 \\
\hline 2 & -16.3005 & 1.0000 & -2025.0146 & 1.0000 & -0.3548 & 0.6385 & $-2.2 \mathrm{e}^{4}$ & 1.0000 \\
\hline 4 & -16.4249 & 1.0000 & -2040.7124 & 1.0000 & -0.3576 & 0.6395 & $-5.1 \mathrm{e}^{5}$ & 1.0000 \\
\hline 8 & -16.6738 & 1.0000 & -2072.1083 & 1.0000 & -0.3630 & 0.6415 & $-6.2 \mathrm{e}^{6}$ & 1.0000 \\
\hline CRPS & & & & & & & & \\
\hline 1 & $2.2 \mathrm{e}^{4}$ & $<0.001$ & $1.4 \mathrm{e}^{07}$ & $<0.001$ & $2.4 \mathrm{e}^{4}$ & $<0.001$ & $2.4 \mathrm{e}^{5}$ & $<0.001$ \\
\hline 2 & $2.1 \mathrm{e}^{4}$ & $<0.001$ & $2.2 \mathrm{e}^{06}$ & $<0.001$ & $2.0 \mathrm{e}^{5}$ & $<0.001$ & $2.4 \mathrm{e}^{4}$ & $<0.001$ \\
\hline 4 & $2.4 \mathrm{e}^{4}$ & $<0.001$ & $2.2 \mathrm{e}^{06}$ & $<0.001$ & $2.0 \mathrm{e}^{5}$ & $<0.001$ & $2.4 \mathrm{e}^{4}$ & $<0.001$ \\
\hline 8 & $2.5 \mathrm{e}^{4}$ & $<0.001$ & $2.4 \mathrm{e}^{06}$ & $<0.001$ & $2.0 \mathrm{e}^{6}$ & $<0.001$ & $2.4 \mathrm{e}^{4}$ & $<0.001$ \\
\hline QuantS & & & & & & & & \\
\hline 1 & $3.5 \mathrm{e}^{5}$ & $<0.001$ & $3.2 \mathrm{e}^{4}$ & $<0.001$ & $-7 e^{5}$ & 1.0000 & $-3.5 e^{6}$ & 1.0000 \\
\hline 2 & $5.2 \mathrm{e}^{6}$ & $<0.001$ & $4.9 \mathrm{e}^{4}$ & $<0.001$ & $-2 \mathrm{e}^{5}$ & 1.0000 & $-8.3 e^{6}$ & 1.0000 \\
\hline 4 & $5.7 \mathrm{e}^{6}$ & $<0.001$ & $4.9 \mathrm{e}^{4}$ & $<0.001$ & $-1.4 \mathrm{e}^{5}$ & 1.0000 & $-9.0 e^{7}$ & 1.0000 \\
\hline 8 & $3.8 \mathrm{e}^{6}$ & $<0.001$ & $4.9 \mathrm{e}^{4}$ & $<0.001$ & $-2 \mathrm{e}^{7}$ & 1.0000 & $-6.4 e^{7}$ & 1.0000 \\
\hline
\end{tabular}

NOTES: This table reports the results of equal predictive ability tests for GSTAR versus AR and MRSTAR models in quarterly US data in growth rates for the testing period from 1982:Q3 to 2013:Q1. All the tests consider density forecasts generated from real data, according to the model estimated in Table 1. In the Amisano-Giacomini test, the GSTAR has the role of $\bar{S}^{f}$ and the benchmark (MR-ST)AR density forecast the role of $\bar{S}^{g}$. Since here LogS has positive orientation, if $t$-statistic is positive, $f$ is preferred; the weight is assumed 1. 
Table 7: Comparison of predictive ability tests of GSTAR versus linear and symmetric models in monthly U.S. data

\begin{tabular}{|c|c|c|c|c|c|c|c|c|}
\hline \multirow{3}{*}{$\begin{array}{r}\text { SERIES } \\
\mathrm{h}\end{array}$} & \multicolumn{4}{|c|}{ MR-STAR vs AR } & \multicolumn{4}{|c|}{ GSTAR vs MR-STAR } \\
\hline & \multicolumn{2}{|c|}{ IIP } & \multicolumn{2}{|c|}{ UN } & \multicolumn{2}{|c|}{ IIP } & \multicolumn{2}{|c|}{ UN } \\
\hline & \multicolumn{8}{|c|}{ Diebold-Mariano } \\
\hline 1 & \multicolumn{2}{|c|}{0.0000} & \multicolumn{2}{|c|}{0.0000} & \multicolumn{2}{|c|}{0.0000} & \multicolumn{2}{|c|}{0.0000} \\
\hline 2 & \multirow{2}{*}{\multicolumn{2}{|c|}{$\begin{array}{l}0.0003 \\
0.0012\end{array}$}} & \multirow{2}{*}{\multicolumn{2}{|c|}{0.0005}} & \multicolumn{2}{|c|}{0.0000} & \multicolumn{2}{|c|}{0.0000} \\
\hline 4 & & & & & \multirow{2}{*}{\multicolumn{2}{|c|}{$\begin{array}{l}0.0000 \\
0.0000\end{array}$}} & \multicolumn{2}{|c|}{0.0000} \\
\hline 12 & & 0.0026 & \multicolumn{2}{|c|}{0.0038} & & & \multicolumn{2}{|c|}{0.0002} \\
\hline $\mathrm{h}$ & \multicolumn{8}{|c|}{ Giacomini-Whight } \\
\hline 1 & \multicolumn{2}{|c|}{0.0000} & \multicolumn{2}{|c|}{0.0000} & \multicolumn{2}{|c|}{0.0000} & \multicolumn{2}{|c|}{0.0000} \\
\hline 2 & 0.00 & & 0.00 & & 0.00 & & 0.00 & \\
\hline 4 & 0.00 & & 0.00 & & 0.00 & & 0.02 & \\
\hline 12 & 0.01 & & 0.01 & & 0.01 & & 0.04 & \\
\hline Scoring Rule & & & & Amis & acomini & & & \\
\hline $\mathrm{h}$ & IIF & & UN & & IIF & & UN & \\
\hline QSR & $t$-statistic & $p$-val & $t$-statistic & $p$-val & $t$-statistic & $p$-val & $t$-statistic & $p$-val \\
\hline 1 & 5.1057 & $<0.001$ & 4.8320 & $<0.001$ & -5.1057 & 1.0000 & 4.2904 & $<0.001$ \\
\hline 2 & 0.3733 & 0.7067 & 4.9038 & $<0.001$ & 0.3733 & 0.3545 & 4.5631 & $<0.001$ \\
\hline 4 & -0.5440 & 0.2150 & 5.1023 & 0.0083 & 0.5440 & 0.2932 & 4.6200 & 0.0250 \\
\hline 12 & 5.0349 & $<0.001$ & 5.7302 & 0.0152 & -5.0349 & 1.0000 & -1.0039 & 0.9231 \\
\hline $\operatorname{LogS}$ & & & & & & & & \\
\hline 1 & 650.28 & 1.0000 & 602.34 & 1.0000 & 649.281 & $<0.001$ & 43.764 & 0.6773 \\
\hline 2 & -192.36 & 0.8899 & -132.48 & 0.7530 & -192.364 & 1.0000 & 30.450 & 0.3502 \\
\hline 4 & $-3.7 e^{3}$ & 1.0000 & $-2.2 e 03$ & 1.0000 & $3.7 e 03$ & $<0.001$ & 12.304 & 0.1339 \\
\hline 12 & -641.28 & 1.0000 & -459.23 & 1.0000 & 641.2803 & $<0.001$ & 12.439 & 0.1010 \\
\hline CRPS & & & & & & & & \\
\hline 1 & -0.41249 & 0.6599 & -0.2995 & 0.3028 & 0.41249 & 0.3400 & -0.3702 & 0.5265 \\
\hline 2 & -1.2273 & 0.1100 & -0.4102 & 0.2504 & -1.2273 & 0.8899 & -0.7894 & 0.2028 \\
\hline 4 & 2.2718 & 0.0116 & -0.1501 & 0.2050 & -2.2718 & 0.9883 & -1.1490 & 0.0922 \\
\hline 12 & -0.4067 & 0.6578 & -0.9501 & 0.5699 & 0.4067 & 0.3421 & -1.4670 & 0.0579 \\
\hline QuantS & & & & & & & & \\
\hline 1 & 7.3000 & 1.0000 & 5.7031 & 0.9940 & -7.3029 & 1.0000 & 4.2499 & 0.3401 \\
\hline 2 & 7.0429 & 1.0000 & 5.2301 & 0.9024 & 7.0429 & 1.0000 & 3.2993 & 0.2370 \\
\hline 4 & 8.7815 & $<0.001$ & 2.1027 & 0.4501 & -8.7815 & 1.0000 & 2.3409 & 0.1502 \\
\hline 12 & 7.1988 & $<0.001$ & 1.8235 & 0.3501 & -7.1988 & 1.0000 & 2.9302 & 0.0829 \\
\hline
\end{tabular}

NOTES: This table reports the results of equal predictive ability tests for GSTAR versus AR and MRSTAR models in monthly US data in growth rates for the testing period from 1982:Q3 to 2013:Q1. All the tests consider density forecasts generated from real data, according to the model estimated in Table 1. In the Amisano-Giacomini test, the GSTAR has the role of $\bar{S}^{f}$ and the benchmark (MR-ST)AR density forecast the role of $\bar{S}^{g}$. Since here LogS has positive orientation, if $t$-statistic is positive, $f$ is preferred; the weight is assumed 1. 


\section{A Appendix}

\section{A.1 Estimation}

Following Leybourne et al. (1998), estimation is done by concentrating the Sum of Square Residuals function with respect to $\boldsymbol{\theta}$ and $\boldsymbol{\phi}$, that is minimizing:

$$
S S R=\sum_{t=1}^{T}\left(y_{t}-\hat{\boldsymbol{\psi}}^{\prime} \mathbf{x}_{\mathbf{t}}^{\prime}\right)^{2}
$$

where:

$$
\hat{\boldsymbol{\psi}}=[\hat{\boldsymbol{\phi}}, \hat{\boldsymbol{\theta}}]=\left(\sum_{t=1}^{T} \mathbf{x}_{\mathbf{t}}^{\prime}(\boldsymbol{\gamma}, \mathbf{c}) \mathbf{x}_{\mathbf{t}}(\boldsymbol{\gamma}, \mathbf{c})\right)^{-1}\left(\sum_{t=1}^{T} \mathbf{x}_{\mathbf{t}}^{\prime}(\boldsymbol{\gamma}, \mathbf{c}) y_{t}\right)
$$

and

$$
\mathbf{x}_{\mathbf{t}}(\hat{\gamma}, \hat{\mathbf{c}})=\left[\mathbf{z}, \mathbf{z}_{\mathbf{t}}^{\prime} G\left(\hat{\gamma}, h\left(\hat{\mathbf{c}}, s_{t}\right)\right]\right.
$$

This is possible because if $\gamma$ and $c$ are known and fixed, the GSTAR model is linear in $\boldsymbol{\theta}$ and $\boldsymbol{\phi}$, which can be easy computed via Conditional OLS (COLS). In a such a way, the nonlinear least square minimization problem, otherwise necessary, more demanding in terms of parameters to estimate and not available in closed-form, reduces to a minimization on three parameters, and is solved via a grid search over $\gamma_{1}, \gamma_{2}, c$. In our illustrations, both $\gamma_{1}$ and $\gamma_{2}$ are generally chosen between a minimum value of -10 and a maximum of 10 with rate 0.25 in the first three examples the grid for parameter $c_{1}$ is the set of values computed between the $10^{\text {th }}$ and $90^{\text {th }}$ percentile of $s_{t}$ with rate computed as the difference of the two and divided for an arbitrarily high number (here, 200). Anyway, this is a only gross rule of thumb and does not pretend to constitute a general indication for application to other data.

\section{A.2 Measures of Predictive Accuracy}

The out-of-sample predictive properties of the estimated models are investigated via rolling forecast experiment, according to which the series $y_{t}$ is divided in a "pre- 
forecast" period (going from time $\{1 \ldots t\}$ ) from which the model is estimated and the $h$-step-ahead forecasts are computed and compared with the "test" period, going from time $\left\{T^{s} \ldots T\right\}$ where $T^{s}=t+h$; this allows to measure $T-T^{s}-h+1$ out-ofsample forecasts. Let denote the corresponding realization of the series as $y_{t}, y_{T}^{s}$ and $y_{T}$, as well as the corresponding density forecasts as $f_{t}, f_{T}^{s}$ and $f_{T}$. Since our interest lies in short-run forecasting we consider $h=\{1,3,6,12\}$. The point predictive performances of the model $j$ are investigated by four different measures: the mean forecast error (MFE), the symmetric mean absolute percentage error (sMAPE), the median relative absolute error (mRAE) and the root mean square forecast error $(\mathrm{RMSFE})^{13}$ :

$$
\begin{gathered}
M F E_{j, h}=\frac{1}{T-h-T^{s}+1} \sum_{t=T^{s}}^{T-h}\left(y_{t+h}-\hat{y}_{t+h \mid t}^{j}\right) \\
s M A P E_{j, h}=\frac{100\left|y_{t+h}-\hat{y}_{t+h}^{j}\right|}{0.5\left(y_{t+h}-\hat{y}_{t+h \mid t}^{j}\right)} \\
m R A E_{j, h}=\frac{\left|y_{t+h}-\hat{y}_{t+h}^{j}\right|}{\left|y_{t+h}-\hat{y}_{t+h}^{(1)}\right|}, \quad \text { with }(1) \text { indexing the benchmark model; } \\
R M S F E_{j, h}=\frac{1}{T-h-T^{s}+1} \sum_{t=T^{s}}^{T-h}\left(y_{t+h}-\hat{y}_{t+h \mid t}^{j}\right)^{2}
\end{gathered}
$$

Differently, the literature on aggregation of density forecasts is instead in a development phase, and focuses on the so called scoring rules (or opinion pools), peculiar functions enabling the forecaster to properly aggregate the set of conditional predictive density as well as more common measures as Mean Square Forecast Error et similia do for point forecasts. Despite their dated origins in statistics, as documented by Gneiting and Raftery (2007), scoring rules are becoming increasingly applied by contemporaneous econometric literature only recently; see, inter alia, Geweke and

\footnotetext{
${ }^{13}$ In particular, sMAPE and mRAE are recommended when the series is known to present volatility effects or skewness, two features often associated to nonlinearity; see the discussion in Tashman (2000).
} 
Amisano (2011). In a similar fashion, concerning about density forecasting, four different scoring rules are used for aggregate the $T-T^{s}-h+1$ predictive densities produced by the same forecasting exercise:

- the logarithmic score $(\log S)$ :

$$
\log _{j, h}=\frac{1}{T-h-T^{s}+1} \sum_{t=T^{s}}^{T-h} \log \hat{f}_{t+h \mid t}^{j}
$$

corresponding to a Kullback-Liebler distance from the true density; models with higher LogS are preferred.

- The quadratic score, somehow the equivalent of the MSFE in point forecasting, is defined as:

$$
Q S R_{j, h}=\frac{1}{T-h-T^{s}+1} \sum_{t=T^{s}}^{T-h} \sum_{k=i}^{K}\left(f_{t+h \mid t}^{j}-d_{k t}\right)^{2}
$$

where $d_{k t}=1$ if $k=t$ and 0 otherwise; models with lower QSR are preferred.

- The (aggregate) continuous-ranked probability (CRPS) score, equivalent to the sMAPE, is defined as:

$$
C R P S_{j, h}=\frac{1}{T-h-T^{s}+1} \sum_{t=T^{s}}^{T-h}\left(\left|f_{t+h}-\hat{f}_{t+h \mid t}^{j}\right|-0.5\left|f_{t+h}-f_{t+h}^{\prime}\right|\right)
$$

where $f$ and $f^{\prime}$ are independent random draws from the predictive density and $f_{t+h \mid t}$ the observed value; models with lower CRPS are preferred.

- Finally, the quantile score (qS) can be obtained if $f_{t+h \mid t}^{j}$ is replaced in (23) by a predictive $\alpha$-level quantile $q_{t+h \mid t}^{\alpha}$ (and the logarithmic function removed); this score is used in risk analysis because it provides information about deviations from the true tail of the distribution. 